\title{
Dynamic viscoplastic granular flows: A persistent challenge in gas-solid fluidization
}

\author{
Kaiqiao Wu, Victor Francia, Marc-Olivier Coppens
}

Centre for Nature Inspired Engineering and Department of Chemical Engineering, University College London, Torrington Place, London WC1E 7JE, United Kingdom, E-mail: m.coppens@ucl.ac.uk, Tel: +44 (0)20 31081126 / +44 (0)20 76797369

\begin{abstract}
Fluidization is a prime example of complex granular flows driven by fluid-solid interactions. The interplay of gravity, particle-particle and fluid-particle forces leads to a rich spectrum of hydrodynamic behavior. A number of complex mathematical formulations exist to describe granular flows. At a macroscopic scale, Eulerian models based on the Kinetic Theory of Granular Flow (KTGF) have been successfully employed to simulate dilute and moderately dense systems, such as circulating fluidized bed reactors. However, their applications to dense flows are challenging, because sustained particle contacts are important. As solid fraction rises, the behavior of granular media responds dramatically to particle properties and changes in concentration. Lacking a coherent transition between formulations of dilute, dense and quasistatic flow behavior, kinetic models are incapable of describing how microstructure emerges and affects the rheology. The behavior of transitional granular flows, such as pulsed fluidized beds, for which the particulate phase transitions between the viscous and plastic regimes, are good reminders of this limitation. In recent years, tremendous effort has been devoted to finding new ways to describe the effects of sustained solids friction and dense flow rheology. This article provides a perspective on this matter from the viewpoint of gas-solid fluidization and discusses advances in describing the dilute-to-dense transition in a continuum framework. Four innovative approaches prevail to extend or supersede the existing kinetic theory: (i) including effective restitution coefficients, (ii) coupling local granular rheological correlations,
\end{abstract}


(iii) introducing rotational granular energy, and (iv) combining non-local laws. While their reliability is still far from that of a Eulerian-Lagrangian approach, they lay a promising foundation for developing a rigorous description of granular media that merges the classical frameworks of continuous fluid and soil mechanics. The progress of continuum formulations does not compete with multi-scale modeling platforms with an applied focus. Ultimately, combining both is a prerequisite to developing new solid stress models that will improve not only the performance of macroscopic models, but also our understanding of granular physics.

\section{Introduction}

Granular flow is a fascinating area of fluid dynamics, however, the physics underpinning the collective behavior of powders is far less understood than that of fluids. Granular media show more complex dynamic behavior than ordinary fluids, due to the dissipation of energy in inelastic collisions and the creation of mesoscopic structures at moderate concentrations. The application of shear stress onto a collection of particles causes the transport of momentum and a proportional shear rate. When particles consolidate, stress can be transported through collective microstructures emerging from sustained particle contacts, leading to a solid-like response where stress induces a proportional elastic deformation or strain. In this transition region, granular matter, like other complex fluids, displays noteworthy dynamic features. Understanding the complex behavior of dense granular matter could unlock the potential to create new processes, materials and devices. Fig.1 illustrates different flow regimes associated with granular flows, and their commonly recognized concentrations.

(Figure 1 to be put here)

In a highly dilute state, the dispersion of a solid phase is purely driven by the surrounding flow field. The particle velocity is a local function of the fluid velocity, and inter-particle collisions are not frequent enough to impart any memory to the solid flow. As a result, many turbulent, 
particle-laden flows, such as pneumatic conveyors, particulate matter in engines, inhalers and aerosols can be reproduced numerically by some sort of Lagrangian tracking framework with two-phase coupling to the fluid, described via a turbulence modeling platform, e.g. Large Eddy Simulations (LES) [1] or Reynolds Averaged Navier-Stokes (RANS) simulations. Stochastic models can estimate the rate of interparticle collisions and study associated processes, such as coalescence. However, as concentration rises, a higher collision rate leads to more energy dissipation. It then becomes necessary to estimate the momentum exchanged within the solid phase, which requires four-way coupled approaches.

In addition to Lagrangian tracking, the emergence of KTGF more than half a century ago provided a rigorous theoretical basis to describe rapid, dilute granular flows as a continuum, by using the corresponding Boltzmann equations $[2,3]$. The KTGF tracks the energy transport and dissipation via a so-called granular temperature, a type of pseudo-energy that quantifies the kinetic energy in the solid fluctuating velocity, as shown in Fig.1a [4]. The classic KTGF assumes the particles to be ideal rigid spheres, frictionless and slightly inelastic, whereby collisions are binary, instantaneous, and uncorrelated. These simplifications allow the particle velocity distribution to be treated as Maxwellian, but limit the application to the study of granular flows in dilute and moderately dense flows.

When particles consolidate further, they enter first the transitional, and then the quasi-static regime. Transitional granular flows are observed experimentally as a local, yet nonlinear correlation between the stress and the rate of deformation, see Fig.1b, while quasi-static flows exhibit a strong time-dependence and non-locality characteristic of solids, which originates from the transport of momentum and stress through a structure of particle chains, see Fig.1c. Researchers in geophysical engineering and soil physics have focused on describing the effects of non-local granular rheology, which permits more physically relevant and accurate descriptions of steady-shear, dense flows [5]. Nevertheless, these closures are not available in 
the traditional context of fluidization. To model dense gas-solid fluidization, the classic KTGF could be coupled with solid frictional stress models, originated from soil mechanics, to account for sustained particle contacts, as shown in Fig.1c. These correlations assume a local and instantaneous coupling between solid volume fraction and frictional stress, and have been derived empirically. As a result, the improvement attained is quite limited, and the classic KTGF-based continuous framework is still deficient in describing dense granular flows.

Beyond continuum models, one could study all regimes of granular flow with fully resolved Lagrangian frameworks, such as Direct Numerical Simulations (DNS) based in finite-volume Lattice Boltzmann (LB) methods [6], or using averaged methods, such as a discrete element method (DEM), to resolve the individual particle motion using Newton's laws and applying a contact model to describe each collision [7]. These methods provide a direct description of the physics by numerically solving the collective behavior of the solid phase, rather than modeling it with constitutive equations, but they are computationally prohibitive for large-scale applications. Several coarse-grained models have been developed to reduce the computational cost. For example, the Particle-in-Cell (PIC) approach combines aspects of both the Eulerian and Lagrangian frameworks [8], resolving the solid flow by tracking a particle cloud to obtain a representative point of mass for multiple particles, but including a collisional stress correlation to include the effect of particle collisions. Similarly, coarse-grained DEM represents the behavior of a group of particles tracked together as a single virtual particle [9], or applying corrections to the forces involved to recreate the behavior of a finer set of particles [10]. The loss of information due to averaging or coarsening, however, ignores scale-dependent mechanisms and the impacts of heterogeneity on the system. J. Li and his colleagues at the Institute for Process Engineering (Chinese Academy of Sciences) have developed new multiscale alternatives to include interactions at mesoscales. The energy-minimization multiscale (EMMS) methodology formulates stability conditions for each flow regime based 
on the principle of "compromise in competition" between gas and solid flow to predict the macroscopic steady-state behavior $[11,12]$. This generic platform, called "mesoscience" by Li, has shown increasing success, leading to a scale-dependent drag law addressing heterogeneity, a two-step correction to KTGF-based models, and a potential multiscale computational paradigm [13]. Nonetheless, even if solid-gas interactions could be properly addressed by EMMS, a correct description of nonlinear solid stress is fundamental to capture system dynamic behavior. A rigorous understanding of the underlaying granular rheology, in particular for transitional and quasi-static flows, is essential to both Eulerian and Lagrangian frameworks.

Obtaining a proper macroscopic description of the transition from dilute to dense regimes represents a historical challenge to the modeling of fluidized beds, where the solid phase varies across a wide range of concentrations. The freeboard, entrainment, cyclone systems and transport lines develop a dilute solid flow; bubbling columns are dominated by moderate concentrations with occasional formation of dense assemblies; whereas spouting beds mainly consist of quasi-static flows. A comprehensive, yet flexible mathematical description has proven very hard. It must capture dense and transitional regimes but also be valid for dilute and moderate flows. For this reason, the KTGF is often taken as the starting point for improving continuous modelling platforms. While classic KTGF-based models could successfully reproduce the overall statistics for a range of operations, they cannot describe the system dynamics as they fail to identify the formation of dense areas. This hinders reliable scale-up and operation and, as a result, substantial efforts have been devoted to improve continuum frameworks that allow the transition towards dense rheology, including effects associated to sustained friction when long particle chains start to form $[14,15]$.

This article discusses continuum formulations from the perspective of fluidization research, moving from the basic description of dilute flows towards an increasing level of complexity until reaching a solid mechanics approach. In Section 2, we discuss challenges associated with 
the continuum description of frictional, dense flows in the context of fluidization. We then illustrate these issues through a dynamic benchmark problem that involves a transitional flow, and evaluate the performance of the various current-state-of-art models in Section 3. Subsequently, in Section 4 we provide our perspective on the state-of-the-art, with opportunities to progress toward a universal continuum framework that is suited to transition across different flow regimes, occurring in different areas of a fluidized bed or during different stages of its operation.

\section{Granular Rheology in Fluidization Technology}

A better understanding of granular flow is critical to improving multiphase chemical reactor performance. Drying, coating, fluid catalytic cracking (FCC) and gasification are examples of granular processes of particular relevance in the chemical, pharmaceutical, food and energy sectors [16]. Fluidization technology is used to create an intensive interphase transport between gases and solids under homogenous mixing [17]. One can classify fluidized beds in dilutephase and dense-phase operations. The solid volume fraction ranges from around 0.01 in a typical riser to 0.50 in a low-velocity bubbling fluidized bed [18]. The flow properties also depend significantly on the size and type of particles. Geldart classified powders into four different groups based on their fluidization behavior [19]. Large and heavy powders tend to spout and form gas channeling, while too small particles easily agglomerate due to their cohesive nature, making fluidization very difficult. Group B powders, widely used in industrial applications, manifest immediate bubbling at incipient fluidization with little bed expansion. When increasing the superficial velocity beyond the minimum fluidization velocity, $U_{\mathrm{mf}}[20]$, the bed transitions from steady bubbling to slugging and, eventually, turbulent fluidization and entrainment. Different designs have been developed to take advantage of the flow properties of each regime.

\subsection{Dilute Gas-Solid Fluidized Systems}


Existing kinetic frameworks have been tremendously useful to reproduce rapid fluidization, whereby one fluidizes particles under a high gas velocity to achieve intensive contact and large interphase transfer rates. In a typical setup, solids are carried out of a bed by high-velocity gas and recirculated back into the system forming a circulating fluidized bed (CFB). The riser often involves a highly turbulent motion forming lean phases to a maximum of $25 \%$ solid fraction [20], but they can also create local dense regions due to cluster formation in the returning pipes. CFB units are often coupled with a stripper and a regenerator, in which the outflowing catalysts can be regenerated to maintain high activity throughout the process. CFBs have a major impact in oil refining through large-scale FCC units for production of high-octane gasoline. In addition to moving beds [21], L-S. Fan and his group at Ohio State University have extensively investigated the application of CFBs in chemical looping processes for $\mathrm{CO}_{2}$ emission controls, associated to fossil fuel combustion and gasification [22, 23], in which the $\mathrm{CO}_{2}$ produced can be sequestered without further separation process [24]. The particulate phase in a CFB riser is typically modelled using a classic KTGF formulation [25] or hybrid approaches [16], because direct Lagrangian tracking is still computationally prohibitive. Nonetheless, the development of high-performance computing (HPC) with a new generation of Exascale architectures promises very large-scale Lagrangian simulations to become realistic.

\subsection{Dense Gas-Solid Fluidized Systems}

Fluidizing Geldart B particles at lower velocities leads to the collapse of the solids and the formation of gas voids. When the velocity is high enough, the voids span across the entire bed, forming a slugging flow. Decreasing the gas velocity further makes the slugs break down into bubbles and the bed consolidates forming a distinct top surface. Bubbling is the manifestation of an intrinsic instability in the exchange of momentum between the gas and particles [26]. As the gas bubbles form and rise, they move the solids up, into their wakes, and drifts cause 
vigorous mixing. Thereby, the overall performance relies on bubble properties, such as size, rise velocity and spatial distribution.

Controlling the flow pattern is difficult, because the bubble interactions are very hard to predict, leading to chaotic behavior and non-uniform gas residence times. Critically, phenomena such as coalescence, channeling and break-up are scale dependent, which causes distinctly different hydrodynamics in industrial units and tremendous challenges in the design and operation of new processes. The case of a plant in Brownsville, TX (USA) in 1950 provides a good illustration of such difficulties: Two commercial Fischer-Tropsch synthesis reactors were built based on experiments in a slugging pilot reactor, only to find out that, at commercial scale, bubble expansion and rise velocity were much higher than expected. The shorter residence time resulted in a 70\% loss in yield [27]. Nowadays, modeling provides us with a wide array of tools to improve reactor design, leading to much tighter quantification of the uncertainty in scale-up, yet we face the same fundamental challenge in lacking ability to reliably control inherently unscalable, nonlinear phenomena.

\subsection{Dynamic Gas-Solid Fluidized Beds in a Transitional Regime: Pulsed Beds}

To tackle some of the described challenges, one can attempt to manipulate the hydrodynamics of fluidized beds using additional degrees of freedom in their design, so as to stabilize the bubbling phenomena before they degenerate into chaos [28]. Various techniques have been proposed to structure fluidized beds $[28,29]$. In particular, pulsation-assisted fluidization has proven to be a useful, non-intrusive way to homogenize a fluidized bed at a relatively low cost [30, 31]. Furthermore, Coppens et al. [29, 32] have demonstrated that pulsation can be used to create a reproducible flow structure, whereby rising bubbles self-organize into a triangular lattice with an intrinsic horizontal wavelength $\lambda$ (Fig. 2). In such a pattern, gas bubbles nucleate at each cycle at alternate positions separated by $1 / 2 \lambda$. 
(Figure 2 to be put here)

This structure only stabilizes at very dense conditions when the suspension oscillates around the minimum fluidization state. Within every oscillation period, the bed is allowed to consolidate for a short period of time, while the flow goes through a minimum. At this point, the existing bubbles rise slowly and maintain their shape, without collapsing or shifting laterally; the solids partially de-fluidize, but still carry the inertia gained during the previous rise in gas flowrate. When the gas velocity starts to increase again, the solids are gradually accelerated upwards, travelling diagonally towards the bubbles located at a higher level. As a result, a rhomboid region forms in between four bubbles (with vertices at alternate bubble positions in consecutive rows), where the solids remain densely packed, thus are far less mobile, as demonstrated in Fig.3. The formation of dense regions compartmentalizes the solid circulation. It also prevents the lateral movement of bubbles and a "catch-up effect", hereby suppressing coalescence and breakage. This leads to a pattern, governed by a single cycle of bubble nucleation, rise and rupture at the bed surface.

Hence, the formation of dense structures and the nucleation of bubbles are intimately related. Fig.3a illustrates this process with a series of images obtained with a high-speed camera (HSC, Photron FASTCAM SA1). Each structure forms in the wake of an existing bubble (the front vertex) when the solids circulate into its wake and form a pyramid pressed onto the bottom air distributor. The bed then collapses and, when the top bubble rises, the presence of the compressive stress in its tail prevents another bubble to be formed at this site, so that, in the next pulse, bubbles appear on both sides. The new bubbles (and associated side vertices) create a new set of pyramids in alternate positions. When the air velocity increases, these new bubbles rise, shaping the original pyramid into a rhomboid structure. During the next pulse, the presence of the two alternate pyramids causes a new bubble (the tail vertex) to form in the tail of the central rhomboid, pushing it upwards. In this way, the formation and disintegration of these 
granular structures synchronize with the creation and motion of the gas bubbles. When the front vertex bubble reaches the surface, the structure becomes an inverted pyramid that is eventually pushed to the sides when the tail vertex bubble reaches the surface.

The solid velocity magnitude contours depicted in Fig.3b were obtained with particle tracking velocimetry (PTV) analysis [33] of selected HSC videos and post-processed with a proper orthogonal decomposition (POD) method to correct particle image velocimetry (PIV) data [34]. These images confirm how regular structures appear and evolve, as well as connect them to the solid flows with diagonal transit lines between the bubbles. Despite some axial mixing of solids through the movement and rupture of the front and tail bubbles, long-range circulation is heavily suppressed. Mixing is less pronounced than under steady-flow operation, but it has now become a much more controlled, local process, which is driven by two characteristic length scales: the bubble size and the pattern wavelength. In such a system, one would expect to have very tight control over contact time and solid mixing, with transport rates that lie in between those in a fixed and a fluidized bed.

(Figure 3 to be put here)

\section{The challenges of modeling pulsed beds in the transition from dilute to dense regime}

From a theoretical point of view, granular flow can be divided into three different regimes [35]. An inertial regime is often associated with a solid volume fraction of $0<\phi<0.49$ and strong agitation [36]. A transitional regime includes the transition from fluid-like to solid-like behavior when $0.49<\phi<\phi_{c}$, where $\phi_{c}$ is defined as the critical packing. Beyond $\phi_{c}$, granular media enter the quasi-static regime, where stresses become completely independent of shear rates. In the pulsating beds described above, the concentration oscillates, causing the particulate phase to transition in both space and time across the inertial and quasi-static regimes. The synchronization of the bubble nucleation and the formation of mesoscopic dense structures 
discussed in Fig.3 indicate that the creation of a bubble pattern is a manifestation of the viscoplastic behavior of the particle assembly. For that reason, this phenomenon is an excellent benchmark to evaluate new theoretical formulations and their ability to reproduce transitional flows. A model capable of reproducing a regular bubble pattern would give a strong indication that it captures the dominant physics across all flow regimes.

\subsection{Modeling a structured bubble flow with Eulerian-Lagrangian approaches}

Not many researchers have studied regular bubble patterns numerically. Kawaguchi et al. [37] and Wang and Rhodes [38] performed 2D simulations and demonstrated that a pulsating gas flow facilitates homogenization. Nevertheless, they could not reproduce the triangular lattice observed experimentally. Our recent work, using a 3D computational domain, shows that reproducing the experimental patterns is possible by making use of standard CFD-DEM models in a quasi-2D bed [39]. Here, one can easily identify the formation of the dense regions described in Fig. 3 in between the wake, the front and the side of rising bubbles. Fig.4 depicts the formation of dense regions with very low mobility in a single array of bubbles, once they are fully formed and rising.

(Figure 4 to be put here)

Fig.5 shows how the gas streamlines curve immediately to avoid the dense central region, using the bubbles as a shortcut to the top. When different bubble arrays coexist in the bed (Fig.5b) the gas penetrates nearby bubbles and shortcuts through the different arrays diagonally. When the gas velocity increases during a pulse, the particles are dragged towards bubbles at higher levels, creating the diagonal transit lines shown in Fig.3, and shaping the central dense regions into the rhomboids shown in Fig. 3 and 4.

(Figure 5 to be put here) 
The qualitative behavior in the simulations is in good agreement with the visual inspection using high-speed cameras. The circulation of solids converging in the wake of a bubble forms a pyramidal region that first conditions the nucleation during the next pulse and then is pushed up to form a rhomboid that prevents the lateral movement of bubbles in the following pulse. In the case of a bed that accommodates multiple rows of bubbles, this structure also limits the axial movement (catch-up) during the following pulses.

Frictional stress is essential to stabilize bubble flow [26]. Ongoing work with CFD-DEM and TFM simulations using different friction factors for the same system indicates that interparticle friction is critical in forming these regions, and thus a structured pattern. Along the same lines, other researchers have started to study the effect of the contact parameters. Bakshi et al.[40] analyzed the flow dynamics in 250 simulations of a scaled-down system, $5 \mathrm{~cm}$ wide and $0.5 \mathrm{~cm}$ thick. They found that the patterns cannot be formed in a bed of either near-elastic, low-friction particles or of highly inelastic, high-friction particles, as shown in Fig.6. This suggests that too low dissipation leads to the formation of large bubbles, which move and rise in the center of the bed, while too high dissipation fails to form a continuous flow of bubbles.

(Figure 6 to be put here)

In summary, recent work illustrates that solid mechanics plays an important role in forming dynamic bubble patterns. A stable flow structure emerges as a result of the alternation between the transitional and quasi-static regimes, whereby particles creep locally around bubbles and pack densely. To maintain this environment, the input energy must be dissipated largely via multiple enduring frictional contacts, whereas the bed still maintains a sufficient level of fluidization. A state-of-the-art Eulerian-Lagrangian approach explicitly tracks and resolves individual particle collisions; therefore, it can easily handle the rheology in different flow regimes without further assumptions and corrections. 


\subsection{Modeling a structured bubble flow with Eulerian-Eulerian approaches}

On the other hand, classical Eulerian-Eulerian frameworks have proven less successful in reproducing dense flows [41]. This is well known when modeling sandy piles, an hourglass, or U-tubes, where enduring friction dominates [16]. Classic KTGF has been derived to analyze rapid, dilute flows of slightly elastic spheres. Their inherent assumption that collisions are frictionless, binary and instantaneous, renders KTGF unable to account for sustained particle contact; thereby, it is limited to describe the inertial regime, where $0<\phi<0.49$.

The state-of-the-art in describing dense flows in fluidization technology relies on coupling the KTGF with separate frictional stress models derived from the critical state theory of soil mechanics, originating from Coulomb's frictional law. These closures include additive contributions due to friction on top of the KTGF kinetic-collisional terms (Eq.1). These frictional models are activated when the solid volume fraction $\phi$ exceeds a frictional packing limit, $\phi_{f}$. The normal frictional stress is modeled solely as a function of solids fraction, coupling with different frictional shear viscosity models:

$$
\begin{aligned}
& P_{\text {tot }}=P_{\text {kinetic-collisional }}+P_{f} \\
& \mu_{\text {tot }}=\mu_{\text {kinetic-collisional }}+\mu_{f} \quad \text { when } \phi>\phi_{f}
\end{aligned}
$$

Johnson and Jackson [42] proposed a semi-empirical equation for the frictional solid pressure, $P_{f}:$

$$
P_{f}=\operatorname{Fr} \frac{\left(\phi-\phi_{f}\right)^{n}}{\left(\phi_{c}-\phi\right)^{p}}
$$

where $F r, n$ and $p$ are the empirical constants. This correlation is often coupled with the frictional shear viscosity model proposed by Coulomb:

$\mu_{f}=\sin \theta$

where $\theta$ is the angle of internal friction of the solids. Schaeffer [43] also derived a frictional shear viscosity model: 
$\mu_{f}=\frac{P_{f} \sin \theta}{2 \sqrt{\mathbf{I}_{2 \mathrm{D}}}}$

Here, $\mathbf{I}_{2 \mathrm{D}}$ is the second invariant of the deviatoric stress tensor. This model is often coupled with the frictional pressure model proposed by Syamlal et al.[44]:

$P_{f}=A\left(\phi-\phi_{f}\right)^{n}$

where $A$ and $n$ are empirical constants; $A=10^{25}, n=10$ and $\phi_{f}=0.59$ are the typical values used.

These widely used frictional stress models are of an empirical or semi-empirical nature, so one must underscore that, under the same conditions, the predicted stress can vary over many orders of magnitude [2]. Besides, they are extremely sensitive to the frictional packing limit, whose definition and selection are still debatable [45]. Given these limitations, it is not surprising that a classic formulation has not been able to reproduce convincingly a transitional flow. Our previous work shows how they can provide a reasonable prediction of other metrics, such as the bubble size [46], shown in Fig.7, while they still fail in recreating the correct dynamics of a structured bubble pattern. A pattern can indeed form occasionally in Eulerian-Eulerian simulations, but it is unstable and, more importantly, incorrect. Bubbles are often seen to originate from the same positions, leading to a square tessellation, which would then be disturbed quickly after several oscillations.

(Figure 7 to be put here)

The work of Bakshi et al. [47] on the formation of bubble patterns using Eulerian-Eulerian approaches in a full 3D domain reveals similar limitations. They show how the dimensionality of the domain affects the structure of the simulated bubble flow (Fig.8), due to the effects of front and rear walls. The authors associate $3 \mathrm{D}$ simulations to more reproducible bubble flows at $f=4 \mathrm{~Hz}$. Nevertheless, the resulting bubble structure is unstable and, as observed in other works, displays an incomplete triangular tessellation, which does not correspond to the experimentally observed pattern (compare Fig.2 and 8). In this context, it is difficult to 
discriminate artifacts arising from a very steep numerical problem, from the correct reproduction of the underlying physics responsible for a pattern. The quantification of the regularity and reproducibility of a bubble structure are important to discern patterns and quantify their stability.

(Figure 8 to be put here)

These works demonstrate that the current formulation of the KTGF, coupled with a critical state yield stress correlation, is insufficiently predictive, because it tends to underestimate the effective shear viscosity and the dissipation of granular energy in the dense areas of the oscillating bed. Consequently, this formulation is unable to track the formation of the pivots described in Fig.3 and it overpredicts the solid circulation. The marked distinction between the two numerical frameworks can be attributed to the lack of a sophisticated model to describe how solid frictional stresses arise.

More advanced constitutive relations have been put forward to describe dense granular flows, some of them rooted in related disciplines, such as geophysical engineering and soil physics, where granular plasticity is more often relevant [48]. Most of these correlations remain casespecific and have not been extended to be generically applicable across all deformation regimes, let alone be part of a formulation compatible with a two-fluid modeling framework. As a result, the classic Eulerian-Eulerian framework based on a simple modification of the KTGF is still considered by many the state-of-the-art in modeling gas-solid fluidized flows, yet it is often utilized without paying sufficient care to the modeling objectives.

\section{Challenges to extend KTGF to dense flows}

This section reviews the limitations of KTGF-based models and potential routes to attain a framework that is more universally applicable to fluidization. However, it must be noted that KTGF has experienced wide success in predicting inertial-flow dominated fluidization. For 
example, Li et al. performed both 2D and 3D KTGF simulations with three differently configured CFB risers, including different cross-sectional geometries and particle feeding directions [25]. Despite the significant quantitative differences between 2D and 3D simulations, pressure gradients and solids fraction were reproduced with good accuracy. Furthermore, the application of the KTGF to fluidized beds at small and well-controlled scales has had notable success in predicting bubble properties. To mention a few examples, Hulme et al. [49] simulated a 2D bubbling bed of Geldart B glass beads at $2 U_{\mathrm{mf}}$, showing quantitative agreement between the calculated and experimental axial profiles of the mean bubble size, measured by X-ray fluoroscopy. Bubble size, rising velocity and even solids circulation patterns and the expansion of bubbling beds of Geldart A and B particles have been captured using KTGF models [50]. Nonetheless, researchers looking at properties of the emulsion phase have voiced more concerning differences. For example, Hernández-Jiménez et al. measured properties using Particle Image Velocimetry (PIV) and Digital Image Analysis (DIA) in a 5mm thick quasi-2D bed fluidized using a small superficial velocity $\left(1.75 \mathrm{U}_{\mathrm{mf}}\right)$ [51]. They found out that the KTGF model overpredicts the solids rising velocity by nearly one order of magnitude, despite good agreement in the bubble properties. As a result, substantial efforts have been made to extend the KTGF to rough and elastic particles, in order to account for more realistic energy dissipation processes $[14,15]$.

Formulating a robust closure for solids stress remains the major handicap of continuum models, in particular for dense granular flows. Historically, the solid stress expression has been closed via a constant solid viscosity evaluated from direct experimental measurements $[3,52,53]$ or an empirical relationship that includes viscosity as an explicit algebraic function of solid volume fraction [54]. These closures are completely local and contain no time dependence, so they meet difficulties when describing dense flows. 
The introduction of the KTGF allowed including weak non-local effects and a certain time evolution in the solid stress, through the transport of pseudo-thermal energy. Nevertheless, momentum transfer is still modeled locally. Efforts have been made to build a better mechanistic understanding of granular rheology and derive a simple, yet physically relevant theory. Comprehensive investigations have been carried out within the French research network, the Groupement De Recherche Milieux Divisés (GDR MiDi), to characterize steady, uniform, dense granular flows in terms of their velocity profiles, density profiles, and velocity fluctuations, in six different geometries for confined shear flows and surface flows [55]. This impressive body of work illustrates the richness and complexity of granular flow, and depicts the development of a very diverse range of velocity profiles under complex geometries. For example, they have shown how a linear or Bagnold solids velocity profile develops in an inclined-plane geometry surface flow, whereas a linear profile with an exponential tail appears in a drum, and a surface flow on top of a heap. In addition, the experimental study by Potapov and Campbell [56] has demonstrated that the ratio of maximum shear to normal stress, which is defined as the internal angle of friction in soil mechanics, is far from constant in hoppers. It has now become clear that one should not expect a soil plasticity based constitutive law alone to be enough to describe the flow behavior in the dense regime [57]. In this difficult context, several new approaches have been put forward to address the changes induced by sustained particle contacts, either in a local or non-local fashion $[5,58]$.

In the following sections, different routes are described to bridge KTGF for dilute and moderate flows with dense and quasi-static flows, in the context of fluidization technology. We discuss four promising mainstream modifications that open the ways to formulate universal solid stress closures. These potential formulations are presented with increasing degrees of complexity, from relatively innocuous modifications to the classic KTGF framework aimed at including the effects of friction in the dissipation of energy (Section 4.1) or the local rheology (Section 
4.2), to the addition of full transport equations to address the transport of rotational energy (Section 4.3) and non-local effects associated to how microstructure affects the transport of momentum (Section 4.4).

\subsection{Friction represented by effective restitution coefficients}

Directly modifying the existing KTGF theory to account for an increasing solid friction effect is a natural first choice to progress into the transitional regime. Jenkins and Zhang [14] have proposed a simple modification of classic KTGF theory for nearly elastic collisions of slightly frictional spheres, which is widely recognized by the community.

Since particles are treated as nearly elastic, the KTGF structure is essentially the same, and the rate of energy dissipation is mostly dependent on the effect of friction at a particle level. Assuming a Maxwellian rotational velocity distribution, the rotational granular temperature can be expressed in terms of the translational temperature balancing the production rate and dissipation rate of the rotational fluctuation energy in collisions. An effective restitution coefficient, $e_{\text {eff, }}$ can then be derived as a function of the normal restitution coefficient, $e$, solid friction, $\mu$, and tangential restitution coefficient, $\beta_{0}$, taking both the presence of inelasticity and friction into account for the total energy loss. When $\mu$ is small but $\mu_{0}$ is not, $e_{\mathrm{eff}}$ can be expressed as follows:

$$
\begin{aligned}
& e_{e f f}=e-\frac{1}{2} a_{1}+\frac{1}{2} a_{2} \frac{b_{1}}{b_{2}} \\
& a_{1} \equiv \frac{\mu}{\mu_{0}}\left[\pi \mu_{0}\left(1-\frac{2}{\pi} \arctan \mu_{0}\right)+\frac{2 \mu_{0}^{2}}{1+\mu_{0}^{2}}\left(1-2 \frac{\mu}{\mu_{0}}\right)\right] \\
& a_{2} \equiv \frac{5 \mu}{2 \mu_{0}}\left[\frac{\pi}{2} \mu_{0}\left(1-\frac{2}{\pi} \arctan \mu_{0}\right)+\frac{\mu_{0}^{2}-\mu_{0}^{4}}{\left(1+\mu_{0}^{2}\right)^{2}}\right] \\
& b_{1} \equiv\left(\frac{\mu}{\mu_{0}}\right)^{2} \frac{\mu_{0}^{2}}{1+\mu_{0}^{2}}
\end{aligned}
$$


$b_{2} \equiv \frac{\mu}{2 \mu_{0}}\left[\frac{\pi}{2} \mu_{0}\left(1-\frac{2}{\pi} \arctan \mu_{0}\right)+\frac{\mu_{0}^{2}}{1+\mu_{0}^{2}}\right]$

$\mu_{0} \equiv \frac{7}{2} \frac{\mu(1+e)}{1+\beta_{0}}$

where $e, \mu$ and $\beta_{0}$ are parameters that can be characterized experimentally. The resulting $e_{\mathrm{eff}}$ can easily be employed under the umbrella of the classical KTGF. The constitutive correlations for stress and flux of fluctuation energy are thereby influenced by friction via the change in granular temperature induced by the additional dissipation. The replacement of $e$ with $e_{\mathrm{eff}}$ aims to capture the decrease of the solid granular temperature and pressure when the interparticle friction coefficient increases, in a dilute regime [59].

Sun and Battaglia [60] implemented this methodology into MFIX-TFM (open source code from the National Energy Technology Laboratory from the USA) [44] to investigate the dynamics and bed expansion of a mono-dispersed bubbling gas-solid fluidized bed and the segregation in a bi-dispersed one, studying a 2D domain. Jenkins and Zhang's model predicts stronger bubbling and a more dilute emulsion phase than the classical KTGF model, reaching better agreement with experimental, time-averaged bed heights. Besides, it was also shown that, while the classical KTGF model overpredicts segregation, Jenkins and Zhang's model provides good agreement with experimental segregation rates. However, other experimental validations reported by Goldschmidt et al. [61] show that the use of a lower $e_{\text {eff }}$ can also be detrimental and lead to artificially vigorous bubble dynamics, which suggests that particle friction could not be accounted for simply by applying a smaller restitution coefficient.

More recently, Jenkins and Berzi [15] further extended the Garzo and Dufty formulation to include an additional length scale into the expression for the rate of collisional energy dissipation, with the purpose of accounting for very inelastic, dissipative collisions. The length is identified with the characteristic length of force chains of particles that are experiencing 
multiple, correlated collisions when the solids approach the critical packing [62]. The use of such "chain length" correction intends to capture the increase in granular temperature and the decrease in shear stress ratio in the transitional regime. However, disparities remain between the predictions of a modified KTGF and DEM simulations for both solid stress and granular temperature [59]. To address the issue of overestimating energy dissipation in dense flow, Duan et al. [63] treated interparticle collisions as finite time processes, deriving a modifier for the collisional energy dissipation rate as a function of characteristic collision time, granular temperature and particle fraction. Compared to Jenkins and Berzi's model, the resulting modified KTGF predicted the trend of granular temperature and solid velocity in better agreement with DEM simulations for non-homogeneous flows up to a solid packing of 0.57 .

\subsection{Friction represented by local rheological principles}

A local rheological law-based approach to model dense granular stress has attracted the attention of the fluidization community, because of its simplicity and sound theoretical foundation. A number of works by the GDR MiDi group [55] have shown that the collective behavior of particles in the transitional regime shares many similarities with classical viscoplastic fluids, such as Bingham fluids. The study proves the existence of local rheology for a variety of configurations, in which the shear stress only depends on local shear rate and solids pressure. Therefore, in principle, one could formulate appropriate constitutive laws to model dense granular flow stresses by extracting this local relationship. In a steady-state homogeneous granular flow, a dimensionless shear rate or inertial number, $I$, can be defined as the ratio of the deformation rate and the rearrangement rate, as shown in Eq.12:

$$
I=\frac{\dot{\gamma} d_{p}}{\sqrt{P / \rho}}
$$


where $P$ is the isotropic solids pressure, $\dot{\gamma}$ is the shear rate, $d_{\mathrm{p}}$ stands for the particle size, and $\rho$ is the particle density. Small values of $I$ represent the quasi-static regime, where the macroscopic deformation is very slow compared to microscopic recovery, whereas a large $I$ corresponds to rapid flow. An effective friction coefficient, $\mu_{\text {eff }}$, can then be defined as:

$$
\mu_{\mathrm{eff}}=\frac{\tau}{P}
$$

where $\tau$ is the solids shear stress. Based upon a number of previous experimental [64] and computational works [65], Jop et al. [58] proposed a new constitutive relationship between the effective friction $\mu_{\text {eff }}$ and $I$, considering static friction effects:

$\mu_{\mathrm{eff}}(I)=\mu_{\mathrm{s}}+\frac{\mu_{2}-\mu_{s}}{1+I_{0} / I}$

where $I_{0}$ is a constant, and $\mu_{2}$ and $\mu_{\mathrm{s}}$ depend on the particle properties and can be measured experimentally. Considering dense granular flow as a form of peculiar non-Newtonian, incompressible fluids, Jop et al. [58] further generalized the stress tensor expressions:

$$
\sigma_{i j}=-P \delta_{i j}+\tau_{i j}
$$

$$
\tau_{i j}=\eta \dot{\gamma}_{i j} \quad \text { with } \eta=\frac{\mu(I) P}{|\dot{\gamma}|}
$$

where $|\dot{\gamma}|$ is the second invariant of the shear rate tensor: $|\dot{\gamma}|=\sqrt{\frac{1}{2} \dot{\gamma}_{i j} \dot{\gamma}_{i j}}$.

The simplicity and successful validation of this rheological law have motivated a number of subsequent computational works. For example, Schneiderbauer et al. [66] coupled this local rheological law with a Eulerian-Eulerian approach to model multiple-spout quasi-2D fluidized beds and a discharging process from a rectangular bin. The authors observed that the local rheological law-based model is able to simulate the flow pattern in the off-spout region correctly, where sustained particle contacts are dominating, and they achieved excellent 
agreement for the discharged rate, with an error below 2.5\%. An earlier formulation developed by Srivastava and Sundaresan [67], using a critical state-based frictional stress model, overpredicted the bed expansion in a single-spout bed, and did not generate the observed relationship between discharge rate and particle diameter. More recently, Farzaneh et al. [68] have studied the solid motion numerically in a 2D dense bubbling fluidized bed of glass beads, and compared local rheological models with state-of-the-art soil mechanics models. The soil mechanics-based models $[43,67]$ were shown to largely underpredict the solid stresses, leading to a completely different flow pattern to the one observed experimentally, whereas much better agreement was provided by the local rheology-based models.

The solid critical packing is essential for a correct rheological formulation. Frictional spheres can jam statically between a random loose packing $(\sim 0.55)$ and a close packing $(\sim 0.634)$ in $3 \mathrm{D}$ domains under different preparation protocols [69]. Several researchers [69, 70] have demonstrated that the critical solid packing can be considered a function of the friction coefficient for soft particles, independently of the normal restitution coefficient. Along those lines, Chialvo et al. $[59,70]$ formulated an extension to the KTGF, based on a comparison of the classical framework at steady state with results of DEM simulations of a Couette flow. Their work extends the original Garzo-Dufty (GD) formulation [71] for dilute flow in a systematic way. Building on previous work, they proposed to couple a new local rheological model with the KTGF to bridge the dense and dilute regimes, along with a series of other corrections to bring the behavior of the modified KTGF in a steady shear flow in agreement with DEM simulations. They identified the following pitfalls and proposed the corresponding corrections:

- Address the critical volume fraction and yields ratio dependence on the friction coefficient.

- Propose a new radial distribution function to be continuous across different regimes and diverge at the critical packing, $\phi_{c}$, specifically for non-equilibrium systems. 
- Decompose solid shear stress to yield stress, estimated using a local rheological model, and inertial stress, calculated using KTGF analysis with a correction factor.

- Modify the energy dissipation term to include an effective restitution coefficient that accounts for solid friction, and a correction factor to reproduce the dense-regime trends with respect to solid packing.

Based on these modifications, they proposed the usual correlation for solids pressure as:

$$
P=\rho H \Theta
$$

Where $\Theta$ is the granular temperature. The solid shear stress, however, is formulated as a combination of a yield term and an inertial term:

$$
\begin{gathered}
\tau=\tau_{\text {yield }}+\tau_{\text {inertial }} \\
\tau_{\text {yield }}=\eta_{s} P \chi \\
\chi=1-\frac{1}{\left(\frac{I_{0} \phi \sqrt{P / \rho}}{\dot{\gamma} d_{p}}\right)^{1.5}+1}
\end{gathered}
$$

where $\eta_{s}$ is the yield stress ratio, and $I_{0}$ is a constant. Following the work of Da Cruz et al. [65] and Jop et al. [58], the blending function $\chi$ combines both inertial and yield stress on the basis of inertial rheology, whereby, at low shear rate the inertial term tends to zero and $\tau$ tends to the value of the yield stress at repose, while at high inertial numbers the contribution of the yield stress drops and the inertial shear stress dominates the system. The solids inertial shear stress itself is given as the GD expression, $\tau_{G D}$, modified with a correction factor, $\delta_{\tau}$ :

$$
\begin{aligned}
& \tau_{\text {inertial }}=\tau_{G D} \delta_{\tau} \\
& \delta_{\tau}=\frac{\alpha}{\beta}+\left(1-\frac{\alpha}{\beta}\right) \chi
\end{aligned}
$$


where $\alpha$ is a constant, and $\beta$ is a transition factor. The numerical results showed reasonable improvement, when compared with DEM simulations over different flow regimes, in terms of granular temperature, pressure and shear stress, as illustrated in Fig. 9.

(Figure 9 to be put here)

It is important to notice that such modifications were derived from the analysis of a single homogeneous system, without the effect of gravity and under steady-state shear granular flow, in which temperature gradients are zero, so that the thermal conductivity effects vanish. The formulation is subject to further debate, and more extensive work would be necessary to make such empirical correlations valid for transient, non-homogeneous dense gas-solid flows. Incorporating the rotational degrees of freedom could somehow mitigate the reliance on empiricism and address the conversion of translational energy to rotational energy directly for a wide range of solid volume fractions, rather than via a constant effective restitution coefficient.

\subsection{Friction induced by rotational granular energy}

From a practical standpoint, the goal is the simplest possible KTGF formulation that can take into account interparticle friction in a physically realistic way. For that reason, the approaches mentioned earlier include solid friction indirectly, by making use of simplifying assumptions or focusing on a macroscopic model. Nonetheless, another natural solution extends the KTGF based on the transport of rotational granular energy. Following the work of Lun et al. [72], Yang et al. [73] constructed a complete set of balance equations and closures for the transport of rough spheres, incorporating both the rotational and translational kinetic granular energy balance on the inertial regime. The proposed set of equations reduces to the classical KTGF formulations in the limit of completely elastic and frictionless spheres $(e \rightarrow 1$ and $\mu \rightarrow 0)$. The translational granular temperature is formulated in the same way as in the original KTGF. The 
authors assumed the bulk rotational velocity to be zero and neglected the change in rotational granular energy caused by interphase interactions, so that transport of rotational granular energy was reduced to consider just the diffusion term and the collisional dissipation term:

$$
\frac{3}{2}\left[\frac{\partial\left(\phi \rho \Theta_{r}\right)}{\partial t}+\nabla\left(\phi \rho \mathbf{V}_{s} \Theta_{r}\right)\right]=\phi \nabla \cdot\left(-\kappa_{r} \nabla \Theta_{r}\right)-\gamma_{r}
$$

where $\Theta_{r}$ is the rotational granular temperature, $\boldsymbol{\kappa}_{r}$ is the thermal conductivity of rotational granular energy, and $\gamma_{r}$ is the dissipation rate of rotational granular energy. The solid stress tensor considers both translational and rotational viscosity:

$$
\tau_{s}=-\left\{\left(\lambda_{s}-\frac{2}{3} \mu_{t s}\right)\left(\nabla \cdot \mathbf{v}_{s}\right) \mathbf{I}+\mu_{t s}\left[\nabla \mathbf{v}_{s}+\left(\nabla \mathbf{v}_{s}\right)^{T}\right]+\mu_{r s}\left[\nabla \mathbf{v}_{s}-\left(\nabla \mathbf{v}_{s}\right)^{T}\right]\right\}
$$

and the rotational viscosity is given as:

$$
\mu_{r s}=-8\left(2 \lambda_{s}+1\right) d_{p} g_{0} \rho \phi A_{1} \sqrt{\frac{\Theta_{t}}{\pi}}
$$

where $A_{l}$ is defined as an integral of trigonometric functions, which can be found in [73].

The authors implemented these correlations to model dense bubbling beds of $1 \mathrm{~mm}$ and $3 \mathrm{~mm}$ glass beads at $3 \mathrm{U}_{\mathrm{mf}}$ to investigate the influence of particle friction. They observed a similar relation between granular temperature and solids fraction to Jenkins and Zhang's models (Eq.611) [14]. Due to the more realistic inclusion of energy dissipation, the granular temperature in both models is shown to be lower than that calculated using the classical KTGF.

Yang et al. [74] also studied the performance of this model against PIV-DIA experimental data and results obtained using a Eulerian-Lagrangian approach in bubbling fluidized beds. They showed that both their model and Jenkins and Zhang's model [14] overpredict the ascending and descending solid velocity in a bed fluidized at a low superficial velocity, $1.7 \mathrm{U}_{\mathrm{mf}}$, while they underpredict it for larger velocities, $\sim 2.3 \mathrm{U}_{\mathrm{mf}}$ (Fig.10). 
(Figure 10 to be put here)

Nonetheless, the extended model reaches better agreement with experimental results than Jenkins and Zhang's model for the time-averaged particle axial velocity and the solid volume fraction. The distribution of translational granular temperatures and the solids circulation agree satisfactorily with the results of Eulerian-Lagrangian simulations, as shown in Fig. 11.

(Figure 11 to be put here)

Compared to the indirect way of describing rotation proposed by Jenkins and Zhang [14], the formulations developed by Yang et al. are promising advances in the inclusion of rotational granular energy. Discrepancies with the solid motion observed in DEM simulations are, however, still prominent, occasionally up to $50 \%$ in terms of time-averaged axial velocity. A major hurdle remains the assumption of a zero mean rotational velocity used in the derivation, which leads to a nearly uniform distribution of rotational granular temperature in the solid phase, which prevents its possible diffusion. DEM simulations, however, show higher rotational energy at the top center of the bed. Finally, Yang et al. also point to the use of simplified boundary conditions that impose a zero gradient of rotational temperature. This constraint impedes the dissipation of granular energy via particle-wall friction, even though wall friction is known to be an essential influence in quasi-2D systems [75].

\subsection{Friction related to the formation of microscopic structures}

In contrast to transitional flows, quasi-static granular flows manifest completely different rheology, which is typified by non-locality, time-dependence or history effects. The rheology of a shear flow is local only if the stress tensor is a function of the shear rate at the same site, whereas any deviation is non-local [48]. The models discussed previously address frictional dissipation by assuming a local correlation between stress and the rate of deformation. A transitional flow has a local, rate-dependent rheology that is captured by the local $I-\mu_{\mathrm{eff}}$ 
rheological law for the inertial and transitional regimes (Eq.14). The latter is obtained from measurements in a steady, spatially uniform shearing environment, such as planar chute flow. However, when granular assemblies consolidate at a slow shear rate (for $I<10^{-3}$, as suggested in $[5,65])$, the granular behavior transitions to a rate-independent response, typical of a solid structure, which leads to non-local effects. For example, in a steady, yet non-uniform, slow, quasi-static flow, the effective friction coefficient, $\mu_{\text {eff, }}$ depends on the system geometry instead of approaching a constant friction coefficient $\mu_{\mathrm{s}}[55,56,76]$. In addition, creeping flows can occur before granules reaching their yield point in the quasi-static regime [55, 77]. These complex features result from long-range transmission of stresses, and cannot be captured by any of the local correlations discussed earlier, such as the $I-\mu_{\text {eff }}$ correlation in transitional granular rheology or Mohr-Coulomb theory $[5,78]$. Therefore, new sources of non-locality or time-dependence beyond those considered in the KTGF framework are required to describe quasi-static flows.

The drastic rheological changes observed in a quasi-static flow are intimately related to the creation of a microscopic solid structure at high solid fractions $[14,79]$ that is commonly neglected in the studies of the inertial regime. Quasi-static flows are characterised by persistent contacts with multiple neighbors, which lead to long-range transport of stress and momentum through an anisotropic (and non-local) force network. The time scales associated to this transport are much smaller than the relaxation time of any plastic rearrangement in the powder. Therefore, in complex configurations, a single kinematic variable, like the solid fraction $\phi$ used in the classic frictional stress models, becomes insufficient to describe the rheology. This brings the question of how non-local effects can be accounted for in a general formulation of granular flow? KTGF only allows for weak non-locality via the granular temperature and its time dependence. More advanced rheological models rooted in granular mechanics have addressed the non-local effects by incorporating an additional state field. In essence, they introduce a 
secondary state variable, independent of the strain rate, but governed via a separate timeevolution equation that describes the change in microstructure.

A common alternative as a non-local measurement of the structure is the coordination number, i.e., the number of particles in contact with each other within a mesoscopic volume. Similar to the classical framework, Aranson and Tsimring [80, 81] decompose the overall stress tensor into a weighted solid- and fluid-like part. The ratio of the two terms is governed by a spacetime order field $\psi=\left\langle Z / Z_{\mathrm{c}}\right\rangle$, where $Z$ and $Z_{\mathrm{c}}$ are the number of particles under persistent contacts and any contacts, respectively. A binary contact is considered persistent when the flow is below the yield condition or the contact duration is longer than a critical collision time. In such a way, $\psi$ is unity when fluid-like behavior vanishes, whereas $\psi$ approaches zero when fluid-like behavior dominates. To close the system, the authors assume that the time-evolution of $\psi$ obeys a generic dispersion equation:

$$
\frac{\partial \psi}{\partial t}+\mathbf{v} \nabla \psi=D \nabla^{2} \psi-F(\psi, \delta)
$$

where $D$ is a diffusion coefficient and $F(\psi, \delta)$ is a derivative of the free energy density that accounts for the relative stability near the transition between solid-like and fluid-like behavior. The control coefficient $\delta$ is a function of the effective friction coefficient:

$\delta=\left(\mu_{\mathrm{eff}}^{2}-\mu_{0}^{2}\right) /\left(\mu_{1}^{2}-\mu_{0}^{2}\right)$

where $\tan ^{-1} \mu_{0}$ and $\tan ^{-1} \mu_{1}$ are the angle of repose and the internal angle, respectively. In subsequent work, Volfson et al. quantify the structure of the constitutive relation and free energy density function by fitting $\psi$ and the stress relation with a simulated thin Couette flow $[82,83]$.

The complete set of constitutive equations yields a different rheology, incorporating a more realistic fluid-solid transition. This approach has been validated on multiple configurations, such as chute flow, Couette flow and a rotational drum [81-83], showing reasonable 
quantitative agreement between the rheology and the velocity profiles expected from analytical solutions with experimental and computational results. Nevertheless, the creeping flow phenomenon is not yet addressed and the numerically fitted correlation is strongly sensitive to the configuration, i.e., 2D or 3D and the chosen contact models.

Other promising non-local constitutive laws for creeping quasi-static flows were proposed by Kamrin [5]. Following the concept of fluidity in emulsions, the authors introduce a so-called granular fluidity, $g$, as a coarse-grained field that governs the non-locality $[84,85]$. The granular fluidity is defined as the ratio of shear rate over the effective friction coefficient, $\mu_{\text {eff. }}$ When $\mu_{\text {eff }}>\mu_{s}$, the granular fluidity reduces to an alternative expression of the local rheology relation:

$$
g=\frac{\dot{\gamma}}{\mu_{\mathrm{eff}}} \rightarrow g_{\mathrm{loc}}=H\left(\mu_{\mathrm{eff}}-\mu_{\mathrm{s}}\right) \frac{\left(\mu_{\mathrm{eff}}-\mu_{\mathrm{s}}\right)}{b \mu_{\mathrm{eff}}} \sqrt{\frac{P}{m}}
$$

$H$ is the Heaviside function, and $b$ is a model parameter. Furthermore, $\xi$ is defined as the internal length scale causing the fluidity to be affected by plastic rearrangements nearby, and is given by:

$$
\xi\left(\mu_{\mathrm{eff}}\right)=\frac{A}{\sqrt{\left|\mu_{\mathrm{s}}-\mu_{\mathrm{eff}}\right|}} d_{p}
$$

where $A$ is a dimensionless scaling constant. A key feature of this formulation is that it may cover all the flow regimes, since $\xi$ vanishes when the flow is above the yield condition. The two spatial dimensions are correlated via a non-local law, expressed as:

$$
\nabla^{2} g=\frac{1}{\xi^{2}}\left(g-g_{l o c}\right)
$$

Simulated flows are consistent with analytical predictions for both a uniform planar chute flow, as well as a non-uniform annual shear flow. For a large $I$ in a steady, annular shear flow, the profiles of $\mu_{\text {eff- }} I$ collapse onto a single curve and align with the local rheological law, whereas, 
in the quasi-static regime, this law can reproduce the effect of the system geometry on the effective friction, see Fig.12a. Besides, the correlation captures a range of boundary-dependent frictional coefficients in quantitative agreement with DEM simulations, see Fig.12b.

In further work, Zhang and Kamrin [86] demonstrated physically that granular fluidity can be regarded as a kinematically observable state variable. According to DEM simulations of three different 3D shear configurations, namely, homogeneous planar shear, planar shear with gravity and chute flows, they found that a universal hyperbola correlates the normalized granular fluidity, $g d / \delta v$, and solid packing, $\phi$. Therefore, granular fluidity can be identified with two kinematic variables: the square root of the granular temperature, $\Theta$, and the solid packing fraction, $\phi$ (Eq.31). This work opens the way to a rigorous, albeit complex introduction of nonlocality in a KTGF framework, linking fluidity with the transport of pseudo-thermal energy, as follows:

$$
g=\frac{\dot{\gamma}}{\mu_{\mathrm{eff}}}=\frac{P}{\eta}=\frac{\rho F_{1}(\phi) \Theta}{\rho d_{p} F_{2}(\phi) \sqrt{\Theta}}=\frac{F_{1}(\phi) \sqrt{\Theta}}{F_{2}(\phi) d_{p}}
$$

where $F_{1}$ and $F_{2}$ can be found from KTGF closures.

(Figure 12 to be put here)

Other alternative state fields have been put forward. For example, Sun and Sundaresan [87] correlate the rheology with the granular microstructure via two kinetic variables: contact or coordination number, $Z$, and the fabric anisotropy tensor, A, i.e., a statistical moment associated with the probability distribution function of a chosen directional vector. In this work, they formulated A against the unit particle center-to-center contact direction vector. They investigated two reversed quasi-static shear flows with DEM, in which the shear direction flips periodically, under either controlled pressure or controlled volume conditions. The solid pressure showed a sudden drop at the reversal of shear direction, followed by a slow recovery to the original value. In contrast, after the shear reversal, the effective friction coefficient 
exhibited a quick change to the same magnitude with an opposite sign. Under this environment, the time evolution of the volume fraction, the effective friction coefficient and the solid pressure obeyed similar profiles as the change of coordination number, $Z$, and anisotropy tensor shear component, $A_{\mathrm{xz}}$, as shown in Fig.13.

(Figure 13 to be put here)

The scaled pressure can be expressed in terms of $Z$ and $\boldsymbol{A}$, as follows:

$$
p d / k=\left(a_{1}+a_{2}|\mathbf{A}|\right)\left(Z-Z_{c}\right)^{2}
$$

where $a_{1}$ and $a_{2}$ are constant coefficients. The coordination number here is defined as $Z=2 N_{d} / N$ with $N_{\mathrm{c}}$ and $N$ the total number of contacts and particles in the contact network, respectively. $Z_{\mathrm{c}}$ is the critical value at jamming. The closure of the effective friction coefficient, $\mu_{\mathrm{eff}}$, is suggested as a function of the following quadratic fitting relation for $\mathbf{A}$.

$$
\mu_{\mathrm{eff}}=\hat{b}_{1}+\hat{b}_{2} \mathbf{A}: \hat{\mathbf{S}}+\hat{b}_{3}(\mathbf{A}: \hat{\mathbf{S}})^{2}
$$

where $b_{1}, b_{2}$ and $b_{3}$ are the modeling parameters; $\hat{\mathbf{S}}=\mathbf{S} /|\mathbf{D}|$, with $\mathbf{S}$ the deviatoric strain rate tensor, and $\mathbf{D}$ the strain rate tensor. To close the model, Sun and Sundaresan also propose the time-evolution of both $Z$ and $\mathbf{A}$ as functions of $\mathbf{A}$ and $\mathbf{D}$. In the full set of equations, the constitutive laws were shown capable of tracking the time evolution of the effective frictional coefficient, fabric, coordination number and volume fraction under both steady and unsteady conditions for a range of particle friction coefficients.

One must admit that from the perspective of fluidization, granules commonly take the form of a dynamic, non-uniform dispersion. Section 4.4 has discussed three promising avenues to describe quasi-static flow by addressing non-locality, from a steady homogeneous state to a heterogeneous state and then a dynamic state, with varying levels of complexity. In principle, combing non-local granular rheological models for the quasi-static regime with local rheology in the framework of the KTGF could lead to a full set of closures universally applicable across all deformation regimes for granular flows. Obviously, it is tempting to construct a generic set 
of continuum equations in the two-fluid framework to tackle common fluidization problems. One must note that the models presented here were derived for fundamental studies and are largely "in the making", but they represent a rigorous way forward.

\section{Conclusions}

A full description of granular flow, even within the context of fluidization technology, has been an open challenge for many years. Kinetic models have been key, but, over the last two decades, their limitations have come to light when dealing with transitional or dense flow systems. Thanks to the development of high-performance computing (HPC) systems, access to a full resolution of granular rheology has improved, but Lagrangian simulations are still prohibitive at commercial scale. Even a scaled-down system, using upcoming exa-scale HPC with CPU×GPU hybrid architectures, would represent a tremendous challenge. From a practical point of view, a macroscopic description of gas-solids suspensions in a Eulerian framework will remain the industry standard in the mid-term for commercial reactor designs. Furthermore, from a theoretical perspective, there is undeniable value in developing deeper understanding of the rheology of powders. The challenge then is to obtain a simple, yet correct way to bridge both, up to now irreconcilable states of granular media: the inertial and quasi-static regimes.

This article has discussed this challenge by describing granular media as a viscoplastic fluid. Some promising approaches to progress towards a complete hydrodynamic formulation of granular flow were highlighted. Advanced frameworks address the role of solid friction in a dense flow with different levels of complexity, namely via a) simple modifications to the KTGF to account for the associated energy dissipation, b) advanced local rheological models, or introducing new transport equations to include c) rotational degrees of freedom or d) a new state field describing the evolution of the granular microstructure and the associated non-local effects in momentum transport. For applications to fluidization, each of these avenues has its own merits, and the adequate level of complexity will depend on the application. 
This work has also introduced the use of pattern formation in pulsating fluidized beds as a way to challenge existing models, because their behavior is intimately related to the transition between inertial and quasi-static regimes. Classic formulations cannot reproduce the creation of a dynamic pattern, because they fail to describe the viscoplasticity of the powder. So far, it is unclear how more advanced formulations would perform, but we propose that confronting modeling approaches with such a dynamic transitional flow is an excellent benchmark to validate and develop better closures and continuum models. A rheological formulation capable of capturing characteristic features of a dynamic bubble pattern, such as its appearance, bubble size, and wavelength, should be robust enough to reproduce transitions between dilute and dense, quasi-static flows. One could expect such a new generation of models to bring about major improvements in the ability to design mostly dense flows, such as spouting or dense bubbling beds.

\section{Acknowledgements}

The authors thank Dr Jonny Higham, U.S. National Energy Technology Laboratory (NETL), for his valuable help in the Particle Tracking Velocimetry (PTV) analysis shown in this work. The research leading to these results has received funding from an EPSRC "Frontier Engineering” Award (EP/K038656/1) and an EPSRC Doctoral Training Award (1528604).

\footnotetext{
List of symbols

$d_{b} \quad$ bubble diameter, $(\mathrm{m}) \quad \dot{\gamma} \quad$ shear rate $(\mathrm{m} / \mathrm{s})$

$d_{p} \quad$ particle diameter, $(\mathrm{m}) \quad \sigma \quad$ solid stress, (Pa)

$e \quad$ particle normal restitution coefficient, $(-) \quad \boldsymbol{\delta}_{i j} \quad$ identity tensor, (-)

$e_{\text {eff }} \quad$ effective restitution coefficient, (-) $\quad \eta \quad$ effective viscosity, $(\mathrm{kg} /(\mathrm{m} \cdot \mathrm{s}))$

$f \quad$ frequency of oscillating flow, $(\mathrm{Hz}) \quad \eta_{\mathrm{s}} \quad$ yield stress ratio, (-)

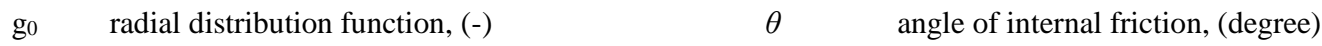

$g \quad$ granular fluidity, $(\mathrm{m} / \mathrm{s}) \quad \lambda \quad$ pattern wavelength, $(\mathrm{cm})$

$I \quad$ inertia number, (-) $\quad \lambda_{s} \quad$ solid bulk viscosity $(\mathrm{kg} /(\mathrm{m} \cdot \mathrm{s}))$

$k \quad$ particle stiffness, $(\mathrm{N} / \mathrm{m}) \quad \mu_{\mathrm{f}} \quad$ frictional shear viscosity, $(\mathrm{kg} /(\mathrm{m} \cdot \mathrm{s}))$
} 


\begin{tabular}{|llll|}
\hline$m$ & particle mass, $(\mathrm{kg})$ & $\mu$ & macroscopic particle frictional coefficient $(-)$ \\
$V_{\mathrm{p}}$ & particle velocity, $(\mathrm{m} / \mathrm{s})$ & $\mu_{\mathrm{tot}}$ & total solid viscosity, $(\mathrm{kg} /(\mathrm{m} \cdot \mathrm{s}))$ \\
$\mathbf{V}_{\mathrm{s}}$ & velocity of solid phase, $(\mathrm{m} / \mathrm{s})$ & $\mu_{\mathrm{s}}$ & static frictional coefficient, $(-)$ \\
$V_{\text {wall }}$ & velocity of inner walls, $(\mathrm{m} / \mathrm{s})$ & $\mu_{\mathrm{eff}}$ & effective frictional coefficient, $(-)$ \\
$P$ & solid pressure, $(\mathrm{Pa})$ & $\mu_{\mathrm{ts}}$ & translational shear viscosity, $(\mathrm{kg} /(\mathrm{m} \cdot \mathrm{s}))$ \\
$P_{\mathrm{f}}$ & frictional solid pressure, $(\mathrm{Pa})$ & $\mu_{\mathrm{rs}}$ & rotational shear viscosity, $(\mathrm{kg} /(\mathrm{m} \cdot \mathrm{s}))$ \\
$P_{\text {wall }}$ & pressure imposed at wall, $(\mathrm{Pa})$ & $\Theta$ & translational granular temperature, $\left(\mathrm{m}^{2} / \mathrm{s}^{2}\right)$ \\
$R$ & rotating drum radius, $(\mathrm{m})$ & $\Theta_{\mathrm{r}}$ & rotational granular temperature, $\left(\mathrm{m}^{2} / \mathrm{s}^{2}\right)$ \\
$t$ & flow time, $(\mathrm{s})$ & $\chi$ & blending factor, $(-)$ \\
$U_{\mathrm{mf}}$ & minimum fluidization velocity, $(\mathrm{m} / \mathrm{s})$ & $\kappa_{\mathrm{r}}$ & pseudo thermal conductivity, $(\mathrm{kg} /(\mathrm{m} \cdot \mathrm{s}))$ \\
$U_{0}$ & superficial gas velocity, $(\mathrm{m} / \mathrm{s})$ & $\rho$ & solids density, $\left(\mathrm{kg} / \mathrm{m}^{3}\right)$ \\
$\mathrm{x}$ & lateral distance from origin, $(\mathrm{m})$ & $\psi$ & fluid order parameter, $(-)$ \\
$\mathrm{x}_{\mathrm{c}}$ & lateral distance from center, $(\mathrm{m})$ & $\tau$ & solid shear stress, $(\mathrm{Pa})$ \\
$Z$ & coordinate number, $(-)$ & $\phi$ & volume fraction of solid phase, $(-)$ \\
$Z_{\mathrm{c}}$ & critical coordinate number, $(-)$ & $\phi_{f}$ & frictional packing of solid phase, $(-)$ \\
& Greek symbols & $\phi_{c}$ & critical packing of solid phase, $(-)$ \\
$\beta_{0}$ & particle tangential restitution coefficient $(-)$ & $\varphi$ & pattern angle, $($ degree $)$ \\
\hline
\end{tabular}

\section{References}

[1] M. Boivin, O. Simonin, K.D. Squires, On the prediction of gas-solid flows with two-way coupling using large eddy simulation, Phys. Fluids 12 (2000) 2080-2090.

[2] B. van Wachem, J.C. Schouten, C.M. van den Bleek, R. Krishna, J. Sinclair, Comparative analysis of CFD models of dense gas-solid systems, AlChE J. 47 (2001) 1035-1051.

[3] T.B. Anderson, R. Jackson, Fluid mechanical description of fluidized beds. Equations of motion, Ind. Eng. Chem. Fundam. 6 (1967) 527-539.

[4] D. Gidaspow, Multiphase flow and fluidization: continuum and kinetic theory descriptions, Academic press, New York, 1994.

[5] K. Kamrin, Nonlinear elasto-plastic model for dense granular flow, Int. J. Plast. 26 (2010) 167 188.

[6] N.G. Deen, J.A.M. Kuipers, Direct Numerical Simulation (DNS) of mass, momentum and heat transfer in dense fluid-particle systems, Curr. Opin. Chem. Eng. 5 (2014) 84-89.

[7] P.A. Cundall, O.D. Strack, A discrete numerical model for granular assemblies, Geotechnique 29 (1979) 47-65.

[8] D. Snider, An incompressible three-dimensional multiphase particle-in-cell model for dense particle flows, J. Comput. Phys. 170 (2001) 523-549.

[9] M. Sakai, S. Koshizuka, Large-scale discrete element modeling in pneumatic conveying, Chem. Eng. Sci. 64 (2009) 533-539.

[10] K. Washino, C.-H. Hsu, T. Kawaguchi, Y. Tsuji, Similarity model for DEM simulation of fluidized bed, Journal of the Society of Powder Technology, Japan 44 (2007) 198-205 in Japanese. [11] J. Li, W. Huang, J. Chen, W. Ge, C. Hou, Mesoscience based on the EMMS principle of compromise in competition, Chem. Eng. J. 333 (2018) 327-335.

[12] J. Li, Exploring the logic and landscape of the knowledge system: multilevel structures, each multiscaled with complexity at the mesoscale, Engineering 2 (2016) 276-285.

[13] J. Li, W. Ge, W. Wang, N. Yang, W. Huang, Focusing on mesoscales: from the energyminimization multiscale model to mesoscience, Curr. Opin. Chem. Eng. 13 (2016) 10-23.

[14] J.T. Jenkins, C. Zhang, Kinetic theory for identical, frictional, nearly elastic spheres, Phys. Fluids 14 (2002) 1228-1235.

[15] J.T. Jenkins, D. Berzi, Dense inclined flows of inelastic spheres: tests of an extension of kinetic theory, Granular Matter 12 (2010) 151-158. 
[16] P. Sreekanth, S. Madhava, J.O.B. Thomas, Computational Gas-Solids Flows and Reacting Systems: Theory, Methods and Practice, IGI Global, Hershey, 2011.

[17] L.-S. Fan, C. Zhu, Principles of gas-solid flows, Cambridge University Press, Cambridge, 2005.

[18] S. Sundaresan, Modeling the hydrodynamics of multiphase flow reactors: current status and challenges, AlChE J. 46 (2000) 1102-1105.

[19] D. Geldart, Types of gas fluidization, Powder Technol. 7 (1973) 285-292.

[20] D. Kunii, O. Levenspiel, Fluidization Engineering, Butterworth-Heinemann, Boston, 2013.

[21] L.-S. Fan, L. Zeng, S. Luo, Chemical-looping technology platform, AlChE J. 61 (2015) 2-22.

[22] L.-S. Fan, L. Zeng, W. Wang, S. Luo, Chemical looping processes for $\mathrm{CO}_{2}$ capture and

carbonaceous fuel conversion-prospect and opportunity, Energ. Environ. Sci. 5 (2012) 7254-7280.

[23] F. Li, H.R. Kim, D. Sridhar, F. Wang, L. Zeng, J. Chen, L.-S. Fan, Syngas Chemical Looping

Gasification Process: Oxygen Carrier Particle Selection and Performance, Energy \& Fuels 23 (2009) 4182-4189.

[24] L.-S. Fan, F. Li, Chemical looping technology and its fossil energy conversion applications, Ind. Eng. Chem. Res. 49 (2010) 10200-10211.

[25] T. Li, S. Pannala, M. Shahnam, CFD simulations of circulating fluidized bed risers, part II, evaluation of differences between 2D and 3D simulations, Powder Technol. 265 (2014) 13-22.

[26] S. Sundaresan, Instabilities in fluidized beds, Annu. Rev. Fluid Mech. 35 (2003) 63-88.

[27] M. Rüdisüli, T.J. Schildhauer, S.M.A. Biollaz, J.R. van Ommen, Scale-up of bubbling fluidized bed reactors - A review, Powder Technol. 217 (2012) 21-38.

[28] J.R. van Ommen, J. Nijenhuis, M.-O. Coppens, Reshaping the structure of fluidized beds, CEP (2009) 49-57.

[29] M.-O. Coppens, J.R. van Ommen, Structuring chaotic fluidized beds, Chem. Eng. J. 96 (2003) $117-124$.

[30] D. Jia, X. Bi, C.J. Lim, S. Sokhansanj, A. Tsutsumi, Biomass drying in a pulsed fluidized bed without inert bed particles, Fuel 186 (2016) 270-284.

[31] A. Akhavan, J.R. van Ommen, J. Nijenhuis, X.S. Wang, M.-O. Coppens, M.J. Rhodes, Improved drying in a pulsation-assisted fluidized bed, Ind. Eng. Chem. Res. 48 (2008) 302-309.

[32] M.-O. Coppens, M.A. Regelink, C.M. van den Bleek, Pulsation induced transition from chaos to periodically ordered patterns in fluidised beds, Proc. of the 4th World Congress on Particle

Technology, Sydney, 2002.

[33] J.E. Higham, W. Brevis, When, what and how image transformation techniques should be used to reduce error in Particle Image Velocimetry data?, Flow Measurement and Instrumentation 66 (2019) $79-85$.

[34] J.E. Higham, W. Brevis, C.J. Keylock, A rapid non-iterative proper orthogonal decomposition based outlier detection and correction for PIV data, Meas. Sci. Technol. 27 (2016) 125303.

[35] I. Goldhirsch, Rapid granular flows, Annu. Rev. Fluid Mech. 35 (2003) 267-293.

[36] S. Torquato, Nearest-neighbor statistics for packings of hard spheres and disks, Phys. Rev. E 51 (1995) 3170.

[37] T. Kawaguchi, A. Miyoshi, T. Tanaka, Y. Tsuji, Discrete particle analysis of 2D pulsating fluidized bed, Proc. of 4th International Conference on Multiphase Flow, New Orleans, 2001.

[38] X. Wang, M. Rhodes, Pulsed fluidization - a DEM study of a fascinating phenomenon, Powder Technol. 159 (2005) 142-149.

[39] K. Wu, L. de Martín, M.-O. Coppens, Pattern formation in pulsed gas-solid fluidized beds-the role of granular solid mechanics, Chem. Eng. J. 329 (2017) 4-14.

[40] A. Bakshi, M. Shahnam, A. Gel, T. Li, C. Altantzis, W. Rogers, A.F. Ghoniem, Comprehensive multivariate sensitivity analysis of CFD-DEM simulations: Critical model parameters and their impact on fluidization hydrodynamics, Powder Technol. 338 (2018) 519-537.

[41] N.G. Deen, M. van Sint Annaland, M.A. van der Hoef, J.A.M. Kuipers, Review of discrete particle modeling of fluidized beds, Chem. Eng. Sci. 62 (2007) 28-44.

[42] P.C. Johnson, R. Jackson, Frictional-collisional constitutive relations for granular materials, with application to plane shearing, J. Fluid Mech. 176 (2006) 67-93.

[43] D.G. Schaeffer, Instability in the evolution equations describing incompressible granular flow, J. Differ. Equations 66 (1987) 19-50. 
[44] M. Syamlal, W. Rogers, T.J. O’Brien, MFIX documentation: Theory guide, National Energy Technology Laboratory, Technical Note DOE/METC-95/1013 and NTIS/DE95000031, 1993.

[45] A. Passalacqua, L. Marmo, A critical comparison of frictional stress models applied to the simulation of bubbling fluidized beds, Chem. Eng. Sci. 64 (2009) 2795-2806.

[46] K. Wu, L. de Martín, L. Mazzei, M.-O. Coppens, Pattern formation in fluidized beds as a tool for model validation: A two-fluid model based study, Powder Technol. 295 (2016) 35-42.

[47] A. Bakshi, C. Altantzis, A. Bershanska, A.K. Stark, A.F. Ghoniem, On the limitations of 2D CFD for thin-rectangular fluidized bed simulations, Powder Technol. 332 (2018) 114-119.

[48] M. Bouzid, A. Izzet, M. Trulsson, E. Clément, P. Claudin, B. Andreotti, Non-local rheology in dense granular flows, The European Physical Journal E 38 (2015) 125.

[49] I. Hulme, E. Clavelle, L. van der Lee, A. Kantzas, CFD modeling and validation of bubble properties for a bubbling fluidized bed, Ind. Eng. Chem. Res. 44 (2005) 4254-4266.

[50] H. Lindborg, M. Lysberg, H.A. Jakobsen, Practical validation of the two-fluid model applied to dense gas-solid flows in fluidized beds, Chem. Eng. Sci. 62 (2007) 5854-5869.

[51] F. Hernández-Jiménez, S. Sánchez-Delgado, A. Gómez-García, A. Acosta-Iborra, Comparison between two-fluid model simulations and particle image analysis \& velocimetry (PIV) results for a two-dimensional gas-solid fluidized bed, Chem. Eng. Sci. 66 (2011) 3753-3772.

[52] J.A.M. Kuipers, K. Van Duin, F. Van Beckum, W. Van Swaaij, A numerical model of gasfluidized beds, Chem. Eng. Sci. 47 (1992) 1913-1924.

[53] Y.P. Tsuo, D. Gidaspow, Computation of flow patterns in circulating fluidized beds, AlChE J. 36 (1990) 885-896.

[54] H. Enwald, E. Peirano, A.E. Almstedt, Eulerian two-phase flow theory applied to fluidization, Int. J. Multiphase Flow 22 (1996) 21-66.

[55] G.D.R. MiDi, On dense granular flows, Eur. Phys. J. E 14 (2004) 341-365.

[56] A.V. Potapov, C.S. Campbell, Computer simulation of hopper flow, Phys. Fluids 8 (1996) 28842894.

[57] Y. Forterre, O. Pouliquen, Flows of Dense Granular Media, Annu. Rev. Fluid Mech. 40 (2008) 124.

[58] P. Jop, Y. Forterre, O. Pouliquen, A constitutive law for dense granular flows, Nature 441 (2006) 727-730.

[59] S. Chialvo, S. Sundaresan, A modified kinetic theory for frictional granular flows in dense and dilute regimes, Phys. Fluids 25 (2013) 070603.

[60] J. Sun, F. Battaglia, Hydrodynamic modeling of particle rotation for segregation in bubbling gasfluidized beds, Chem. Eng. Sci. 61 (2006) 1470-1479.

[61] M.J.V. Goldschmidt, J.A.M. Kuipers, W.P.M. van Swaaij, Hydrodynamic modelling of dense gas-fluidised beds using the kinetic theory of granular flow: effect of coefficient of restitution on bed dynamics, Chem. Eng. Sci. 56 (2001) 571-578.

[62] J. Jenkins, D. Berzi, Dense, collisional, shearing flows of compliant spheres, EPJ Web of Conferences 140 (2017) 01004.

[63] Y. Duan, Z.-G. Feng, E.E. Michaelides, S. Mao, Modified kinetic theory applied to the shear flows of granular materials, Phys. Fluids 29 (2017) 043302.

[64] O. Pouliquen, Y. Forterre, Friction law for dense granular flows: application to the motion of a mass down a rough inclined plane, J. Fluid Mech. 453 (2002) 133-151.

[65] F. Da Cruz, S. Emam, M. Prochnow, J.-N. Roux, F. Chevoir, Rheophysics of dense granular materials: Discrete simulation of plane shear flows, Phys. Rev. E 72 (2005) 021309.

[66] S. Schneiderbauer, A. Aigner, S. Pirker, A comprehensive frictional-kinetic model for gasparticle flows: Analysis of fluidized and moving bed regimes, Chem. Eng. Sci. 80 (2012) 279-292. [67] A. Srivastava, S. Sundaresan, Analysis of a frictional-kinetic model for gas-particle flow, Powder Technol. 129 (2003) 72-85.

[68] M. Farzaneh, A.-E. Almstedt, F. Johnsson, D. Pallarès, S. Sasic, The crucial role of frictional stress models for simulation of bubbling fluidized beds, Powder Technol. 270 (2015) 68-82.

[69] C. Song, P. Wang, H.A. Makse, A phase diagram for jammed matter, Nature 453 (2008) 629. [70] S. Chialvo, J. Sun, S. Sundaresan, Bridging the rheology of granular flows in three regimes, Phys. Rev. E 85 (2012) 021305. 
[71] V. Garzó, J.W. Dufty, Dense fluid transport for inelastic hard spheres, Phys. Rev. E 59 (1999) 5895-5911.

[72] C. Lun, S.B. Savage, D. Jeffrey, N. Chepurniy, Kinetic theories for granular flow: inelastic particles in Couette flow and slightly inelastic particles in a general flowfield, J. Fluid Mech. 140 (1984) 223-256.

[73] L. Yang, J.T. Padding, J.A.M. Kuipers, Modification of kinetic theory of granular flow for frictional spheres, Part I: Two-fluid model derivation and numerical implementation, Chem. Eng. Sci. 152 (2016) 767-782.

[74] L. Yang, J.T. Padding, J.A.M. Kuipers, Modification of kinetic theory of granular flow for frictional spheres, part II: Model validation, Chem. Eng. Sci. 152 (2016) 783-794.

[75] F. Hernández-Jiménez, J. Sánchez-Prieto, E. Cano-Pleite, L.M. Garcia-Gutierrez, A. AcostaIborra, Development of an empirical wall-friction model for 2D simulations of pseudo-2D bubbling fluidized beds, Adv. Powder Technol. 27 (2016) 521-530.

[76] T.S. Komatsu, S. Inagaki, N. Nakagawa, S. Nasuno, Creep Motion in a Granular Pile Exhibiting Steady Surface Flow, Phys. Rev. Lett. 86 (2001) 1757-1760.

[77] N. Taberlet, P. Richard, A. Valance, W. Losert, J.M. Pasini, J.T. Jenkins, R. Delannay, Superstable Granular Heap in a Thin Channel, Phys. Rev. Lett. 91 (2003) 264301.

[78] K. Kamrin, G. Koval, Nonlocal Constitutive Relation for Steady Granular Flow, Phys. Rev. Lett. 108 (2012) 178301.

[79] Q. Zhang, K. Kamrin, A Microscopic Description of the Granular Fluidity Field in Nonlocal Flow Modeling, Phys. Rev. Lett. 118 (2017) 058001.

[80] I.S. Aranson, L.S. Tsimring, Continuum description of avalanches in granular media, Phys. Rev. E 64 (2001) 020301.

[81] I.S. Aranson, L.S. Tsimring, Continuum theory of partially fluidized granular flows, Phys. Rev. E 65 (2002) 061303.

[82] D. Volfson, L.S. Tsimring, I.S. Aranson, Order Parameter Description of Stationary Partially Fluidized Shear Granular Flows, Phys. Rev. Lett. 90 (2003) 254301.

[83] D. Volfson, L.S. Tsimring, I.S. Aranson, Partially fluidized shear granular flows: Continuum theory and molecular dynamics simulations, Phys. Rev. E 68 (2003) 021301.

[84] K. Kamrin, D.L. Henann, Nonlocal modeling of granular flows down inclines, Soft Matter 11 (2015) 179-185.

[85] D.L. Henann, K. Kamrin, A predictive, size-dependent continuum model for dense granular flows, Proceedings of the National Academy of Sciences 110 (2013) 6730-6735.

[86] Q. Zhang, K. Kamrin, Microscopic Description of the Granular Fluidity Field in Nonlocal Flow Modeling, Phys. Rev. Lett. 118 (2017) 058001.

[87] J. Sun, S. Sundaresan, A constitutive model with microstructure evolution for flow of rateindependent granular materials, J. Fluid Mech. 682 (2011) 590-616. 
(a)

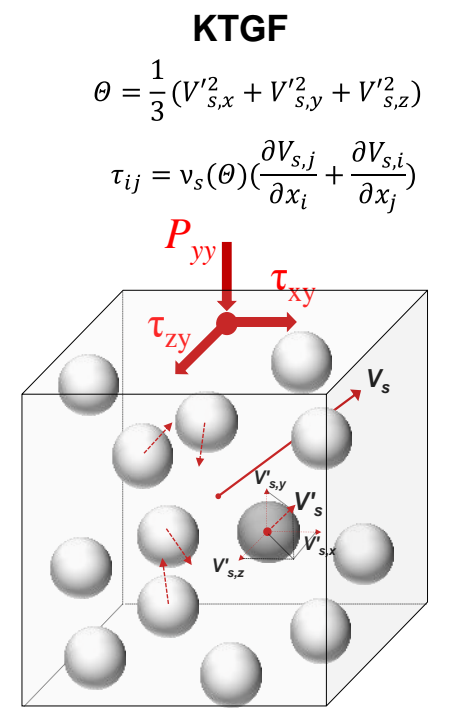

Inertial Regime

$0<\phi<0.49$ (b)

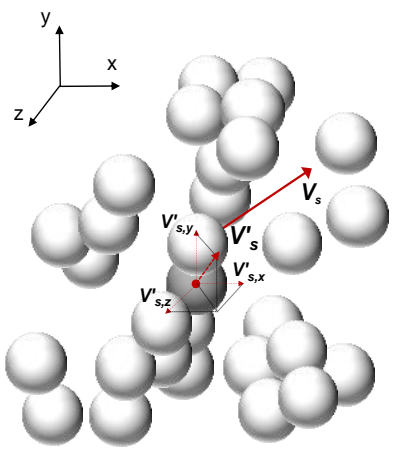

Transitional Regime

$0.49 \leq \phi<\phi_{c}$ (c) Soil Mechanics

$\tau=f(\theta) P$

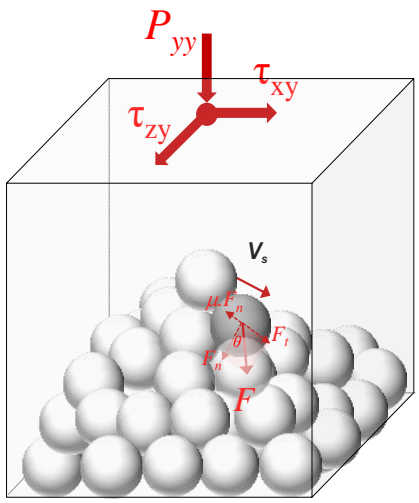

Quasi-Static Regime $\phi \geq \phi_{c}$

Figure 1: Schematic depiction of solid behavior in the (a) inertial regime, (b) transitional regime, and (c) quasi-static regime, with associated modelling methods. 

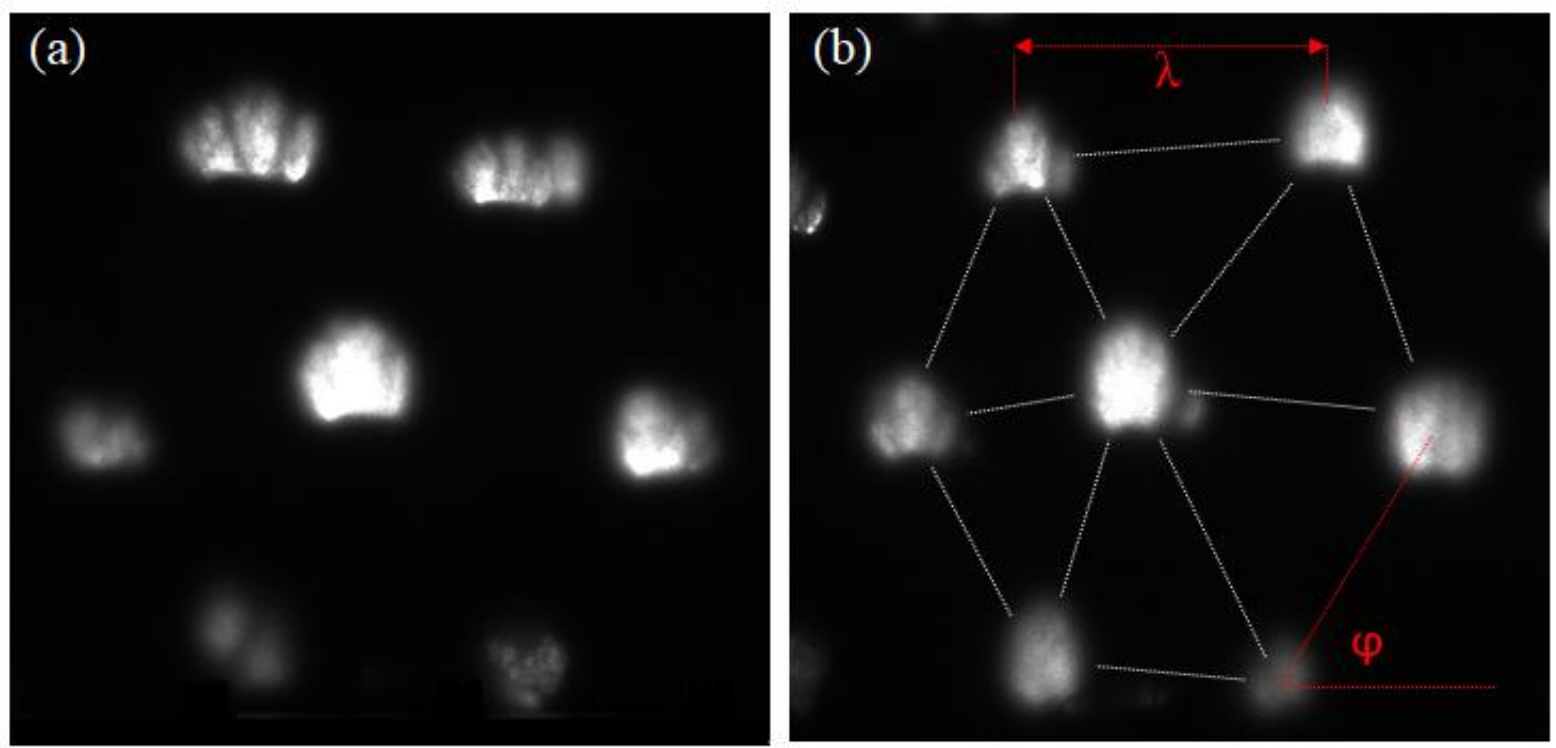

Figure 2: Example of experimentally observed, dynamic bubble patterns in a quasi-2D bed of Geldart B particles, induced by pulsating the gas flow. Snapshots shown in (a) and (b) are separated by two periods of gas pulsation. 



(a)HSC

$\boldsymbol{t}$

(b)PTV

Figure 3: (a) Snapshots of the flow pattern in a dynamically structured pulsed bed, captured using a high-speed camera, and (b) corresponding computation of the solids velocity contours through PTV analysis. The color stands for the scale of the solid velocity, where red to blue depicts decreasing velocity. 


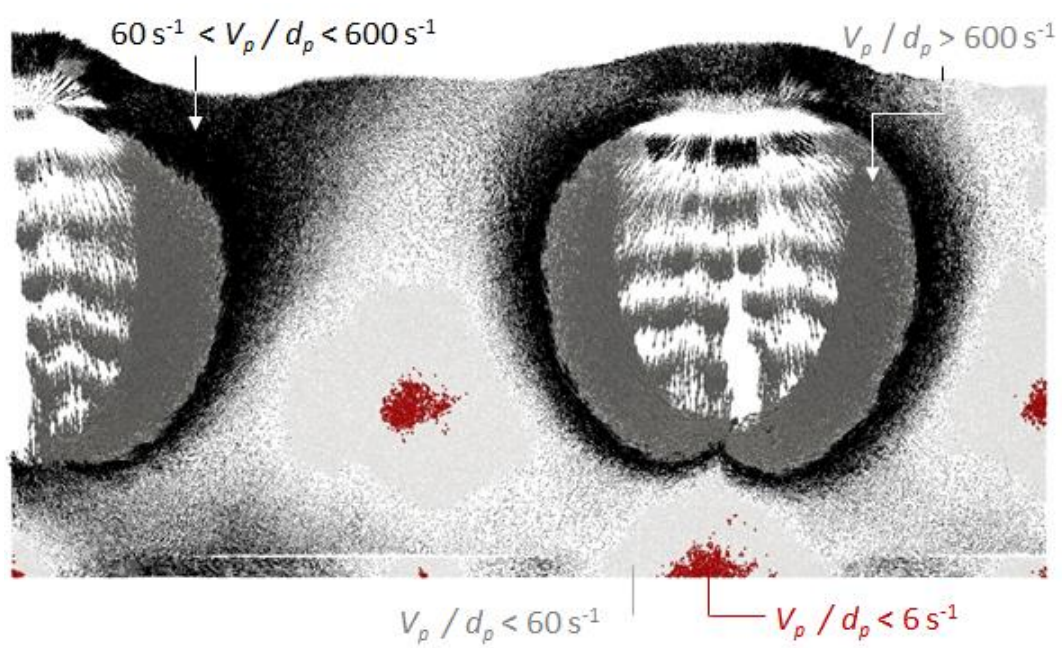

Figure 4: Illustration of the dense areas in a structured fluidized bed. Quiver plot from CFDDEM simulations [39]. The marked area shows locked regions, grey: $V_{\mathrm{p}} / d_{\mathrm{p}}<60 \mathrm{~s}^{-1}$, red: $V_{\mathrm{p}} / d_{\mathrm{p}}$ $<6 \mathrm{~s}^{-1}$. The bed has a solid velocity $60 \mathrm{~s}^{-1}<V_{\mathrm{p}} / d_{\mathrm{p}}<600 \mathrm{~s}^{-1}$; the arrows in the region nearby the bubble, $V_{\mathrm{p}} / d_{\mathrm{p}}>600 \mathrm{~s}^{-1}$, are scaled down by $50 \%$. 
(a)
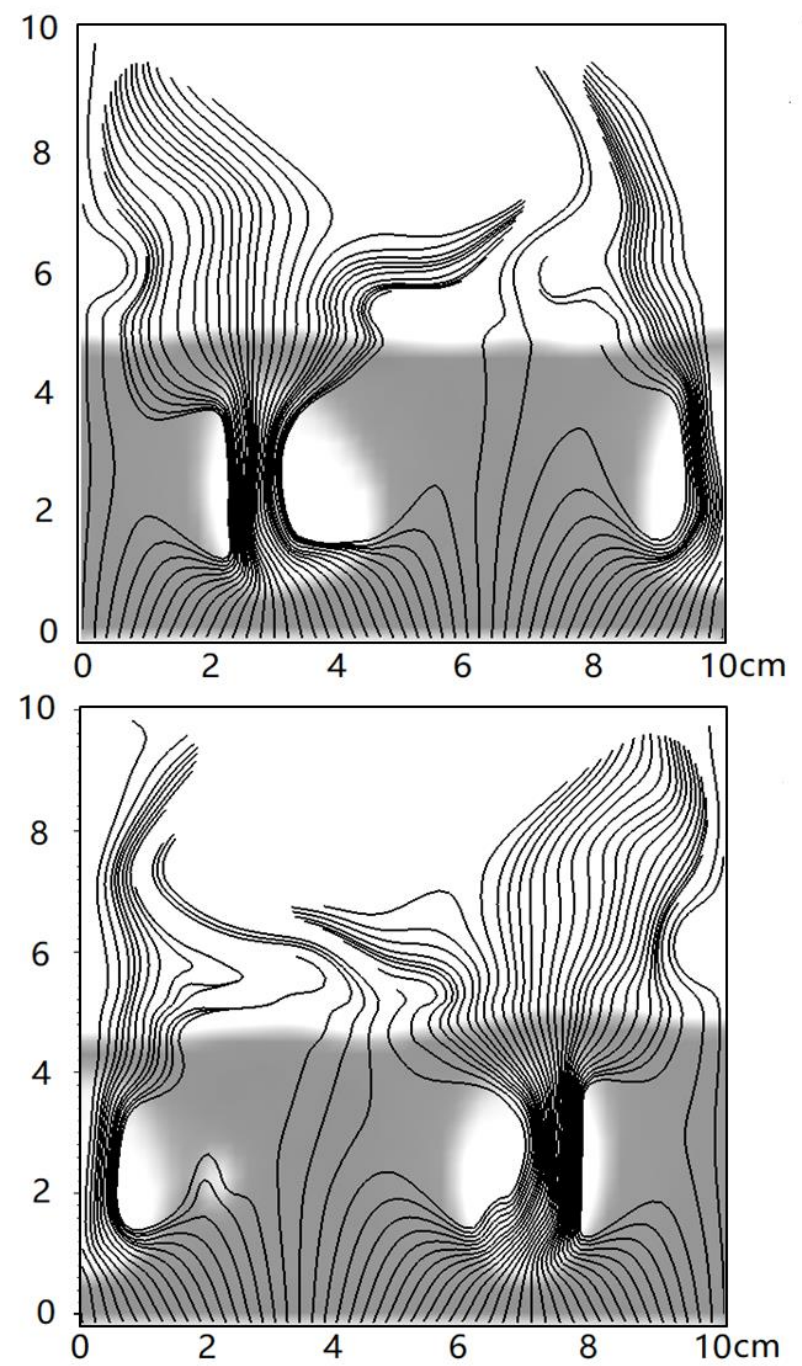

(b)
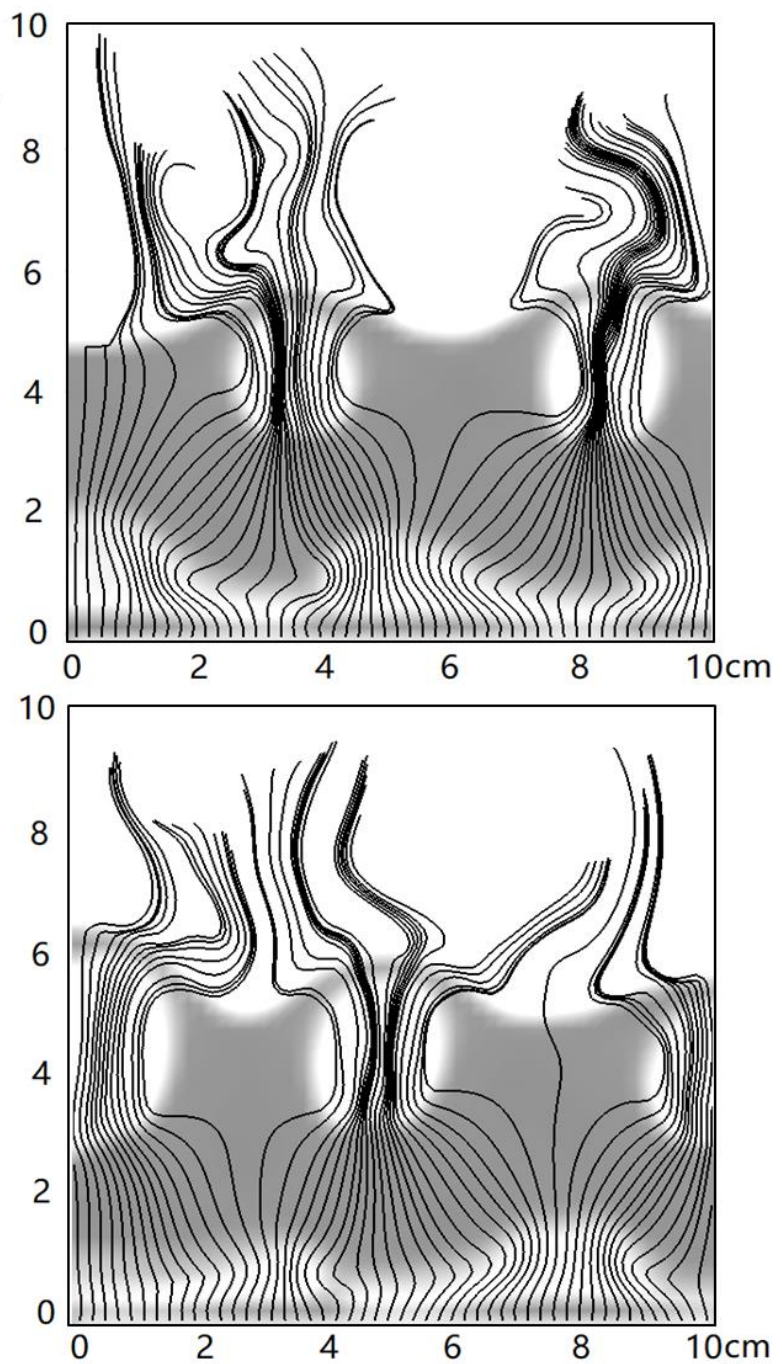

Figure 5: Simulated, patterned flow of bubbles in quasi-2D pulsed beds. The black lines represent the gas streamlines. Conditions: $238 \mu \mathrm{m}$ glass beads; static bed height $4.5 \mathrm{~cm}$ in a domain of $10 \times 10 \times 0.2 \mathrm{~cm} . \mathrm{U}_{0}(\mathrm{~m} / \mathrm{s})=0.108+0.0877 \sin (2 \pi f t)$ with $(\mathrm{a}) f=5 \mathrm{~Hz}$, and (b) $f=7 \mathrm{~Hz}$. 


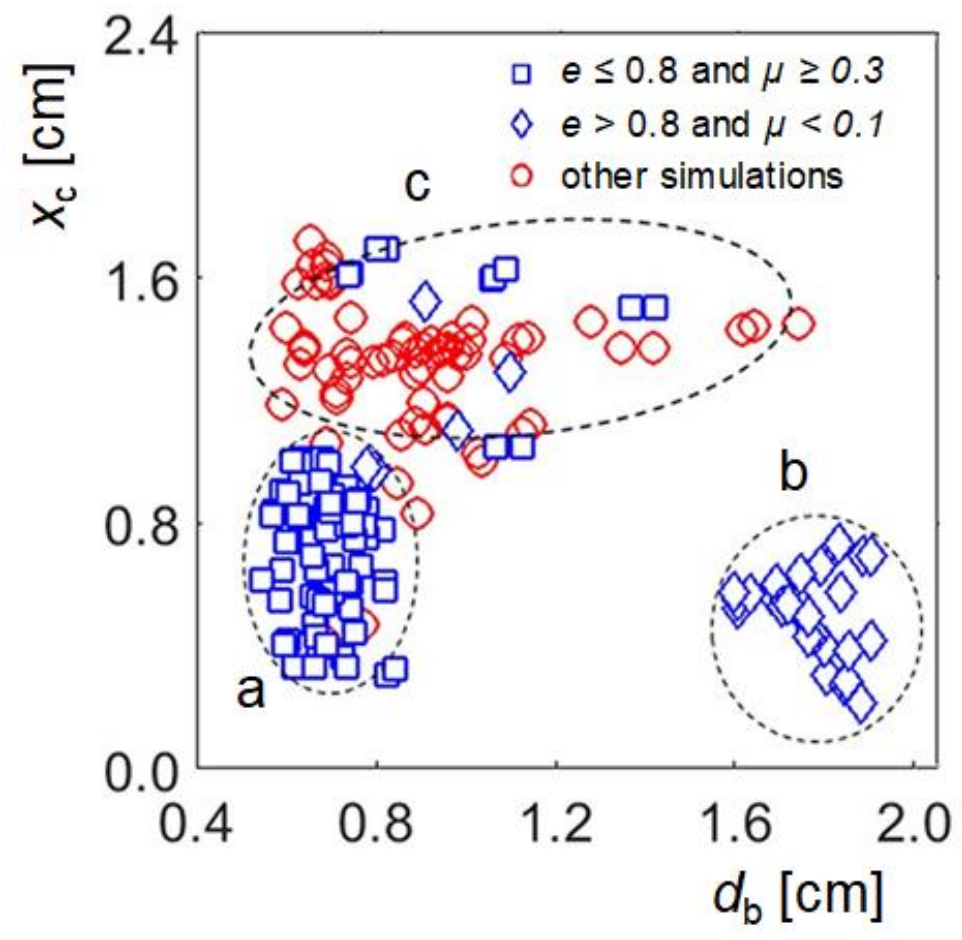

Figure 6: Simulation of small-scale pulsating fluidized bed encompassing one bubble. Average bubble diameter, $d_{\mathrm{b}}$, versus lateral bubble position from the bed center, $x_{\mathrm{c}}$. Here, $e$ and $\mu$ are normal restitution and friction coefficients, respectively. Domains a and $\mathrm{b}$ encompass simulations without any discernible pattern; domain c corresponds to simulations where the position of the bubble nucleation alternates between left and right positions. Static bed height is $3.8 \mathrm{~cm}$; bed cross-section is $5 \times 0.5 \mathrm{~cm} ; \mathrm{U}_{0} / \mathrm{U}_{\mathrm{m}}=1.3+0.7 \sin (2 \pi f t)$. The figure is reprinted with permission from [40]. 

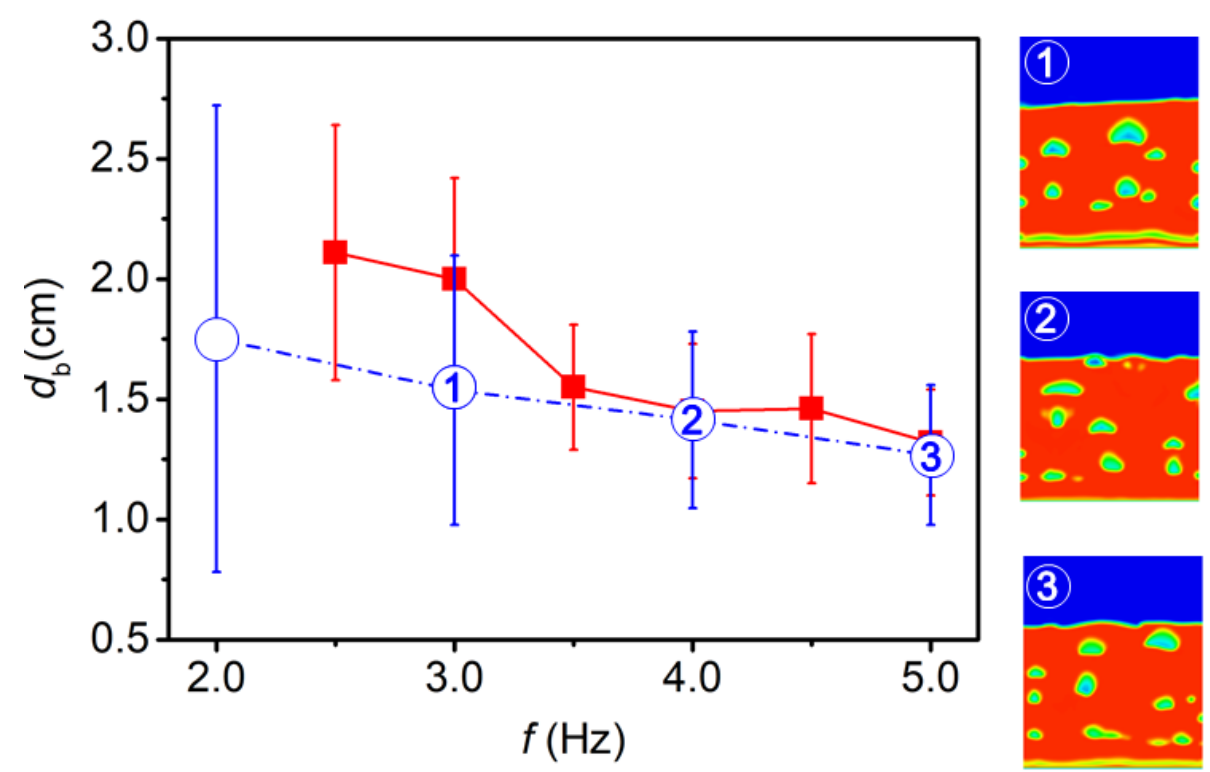

Figure 7: Variation of bubble size with pulse frequency in Eulerian-Eulerian simulations of a 2D pulsed fluidized bed. The error bars represent the standard deviation in the bubble size distribution. The filled symbols stand for the experimental measurements, while the open symbols represent the computational results. The snapshots correspond to $f=3,4$ and $5 \mathrm{~Hz}$. Conditions: $360 \mu \mathrm{m}$ glass beads; bed width $40 \mathrm{~cm}$; static bed height $40 \mathrm{~cm} ; \quad U_{0} / U_{\mathrm{mf}}=1.4+$ $0.4 \sin (2 \pi f t)$. The figure is reprinted with permission from [46]. 
(a)
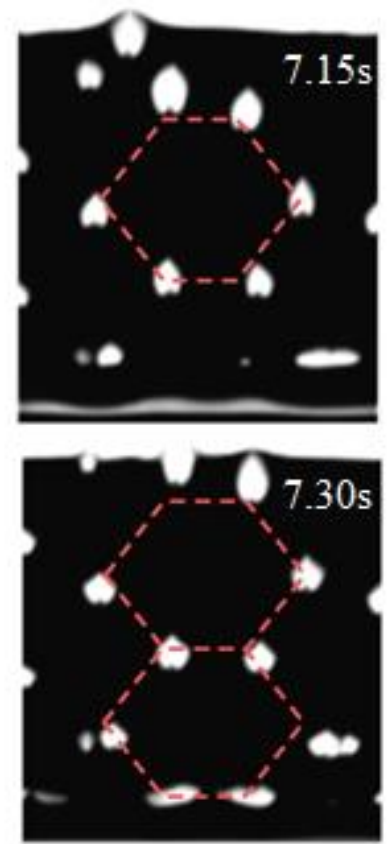

(b)
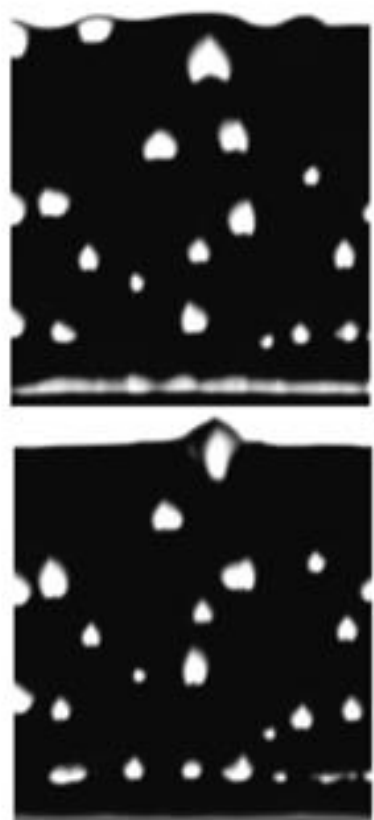

Figure 8: Snapshots of Eulerian-Eulerian simulations of a 2D oscillating bed for (a) 3D and (b) 2D configuration. Conditions: $360 \mu \mathrm{m}$ glass beads; static bed height $40 \mathrm{~cm}$; bed thickness $1.5 \mathrm{~cm} ; U_{0} / U_{\mathrm{mf}}=1.3+0.5 \sin (2 \pi f t)$ with $f=4 \mathrm{~Hz}$. The figure is reprinted with permission from [47]. 

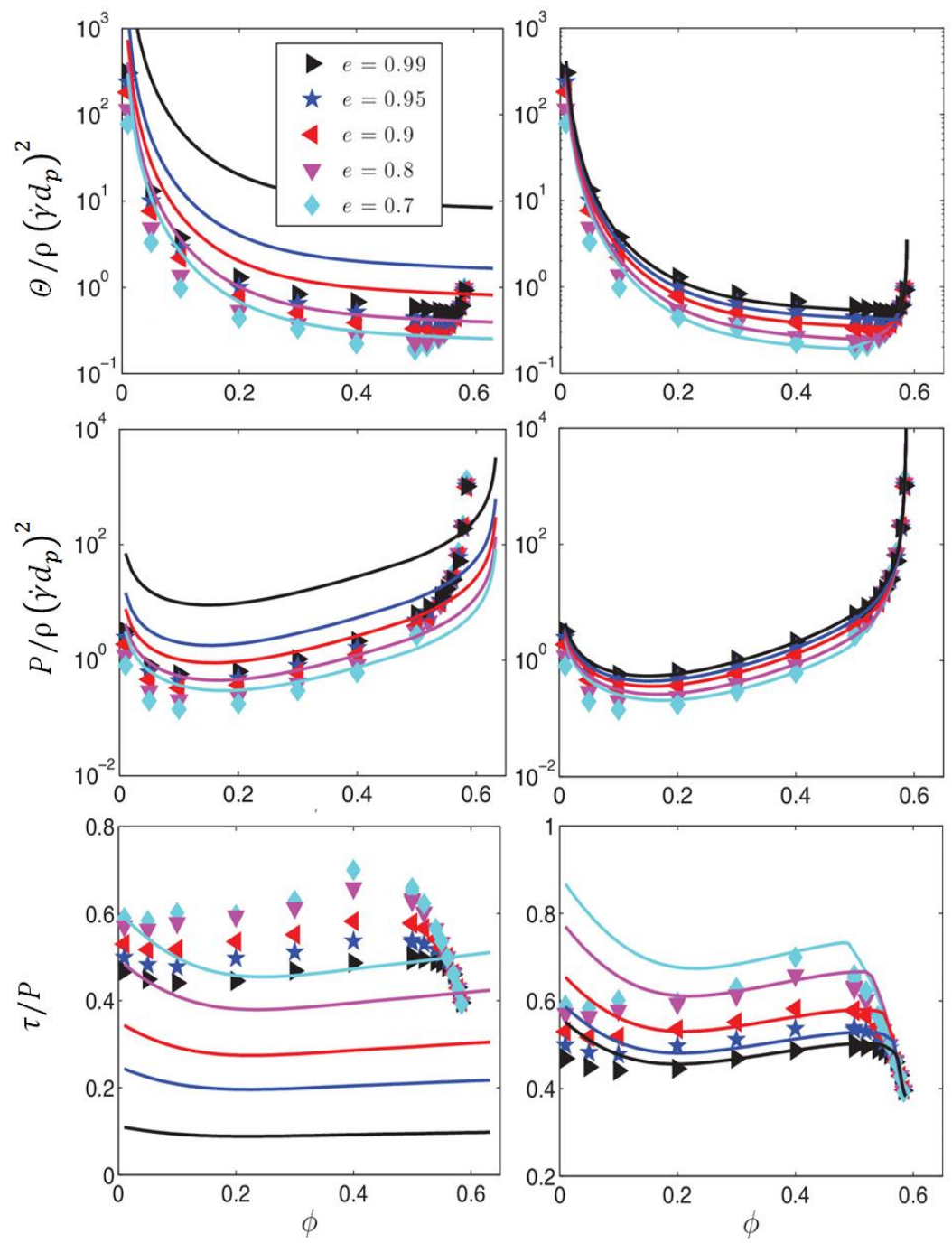

(a) Garzo \& Dufty [71]

(b) Chialvo \& Sundaresan [59]

Figure 9: Rheological corrections to KTGF. Comparison of the Garzo-Dufty KTGF and the modified KTGF by Chialvo and Sundaresan with DEM for a steady shear flow. Granular temperature, pressure and shear stress ratio versus volume fraction are shown for the steady shear flows of $530 \mu \mathrm{m}$ glass beads, with $\mu=0.5$ and different restitution coefficients. The figure is reprinted with permission from [59]. 

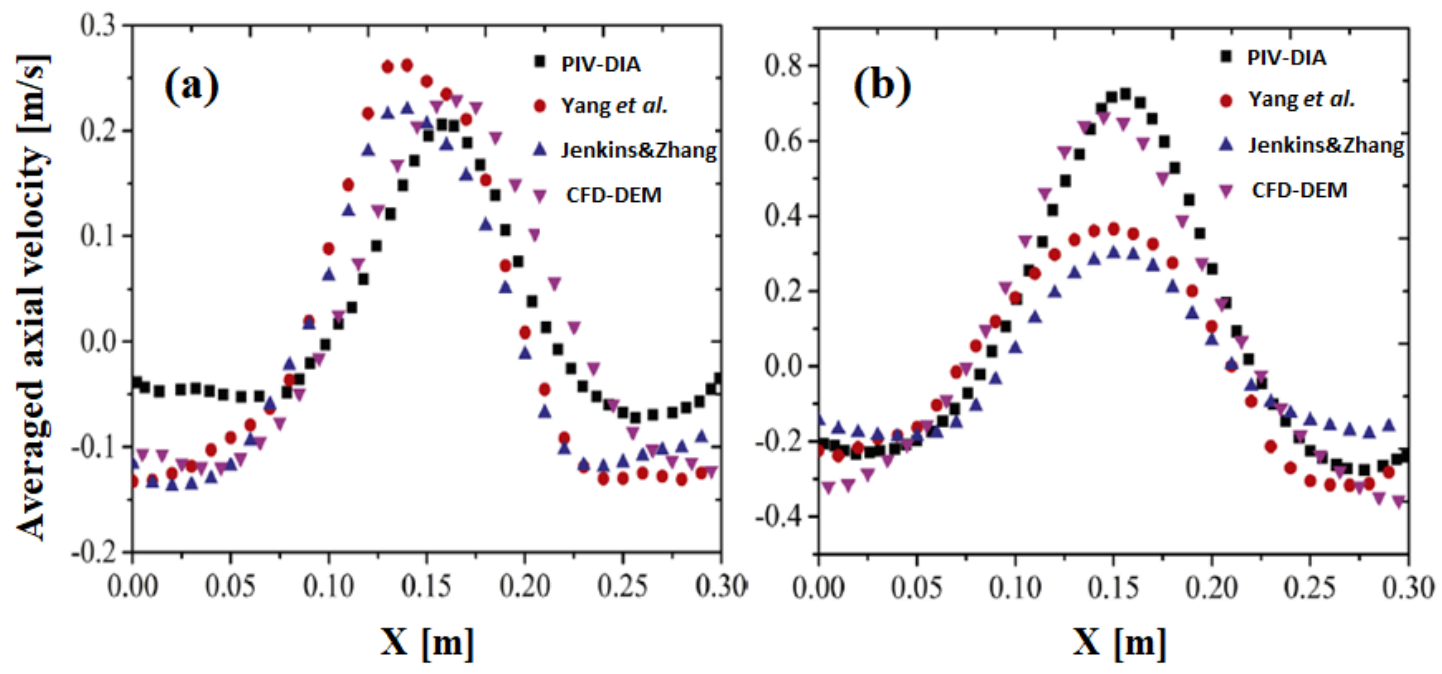

Figure 10: Rotational energy corrections to the KTGF framework. Comparison of the timeaveraged axial particle velocity using PIV-DIA with simulations at a height of $20 \mathrm{~cm}$, for (a) $\mathrm{U}_{0}=2.35 \mathrm{~m} / \mathrm{s}$ and (b) $\mathrm{U}_{0}=3.28 \mathrm{~m} / \mathrm{s}$, with $3 \mathrm{~mm}$ glass beads at a $30 \mathrm{~cm}$ static bed height. The figure is reprinted with permission from [74]. 
(a)

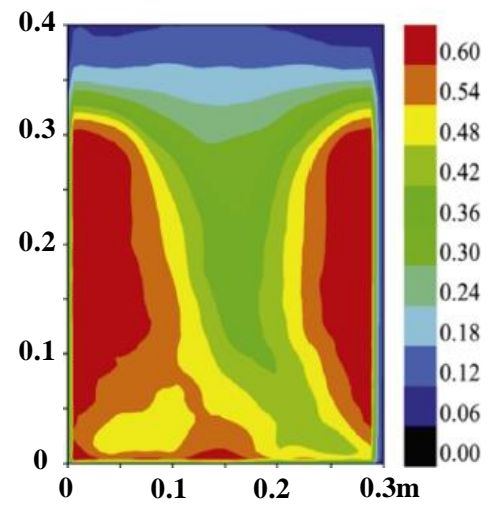

(b)

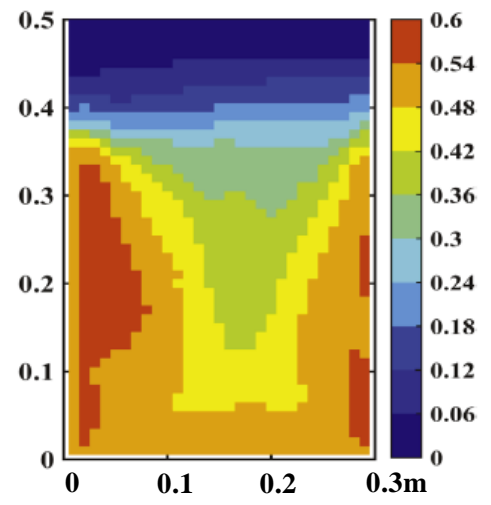

(c) Solid fraction(-)

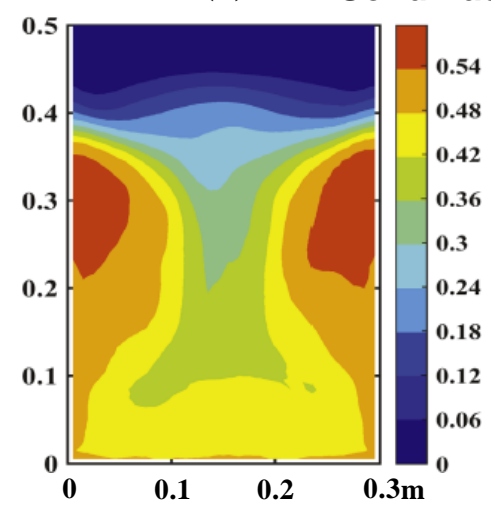

Figure 11: Rotational energy corrections to the KTGF framework. Comparison of timeaveraged solids volume fraction for (a) PIV-DIA, (b) Eulerian-Lagrangian simulations and (c) Eulerian-Eulerian simulations, using the modified KTGF models, with $3 \mathrm{~mm}$ glass beads at a $30 \mathrm{~cm}$ static bed height. $\mathrm{U}_{0}=2.35 \mathrm{~m} / \mathrm{s}$. The figure is reprinted with permission from [74]. 
(a)

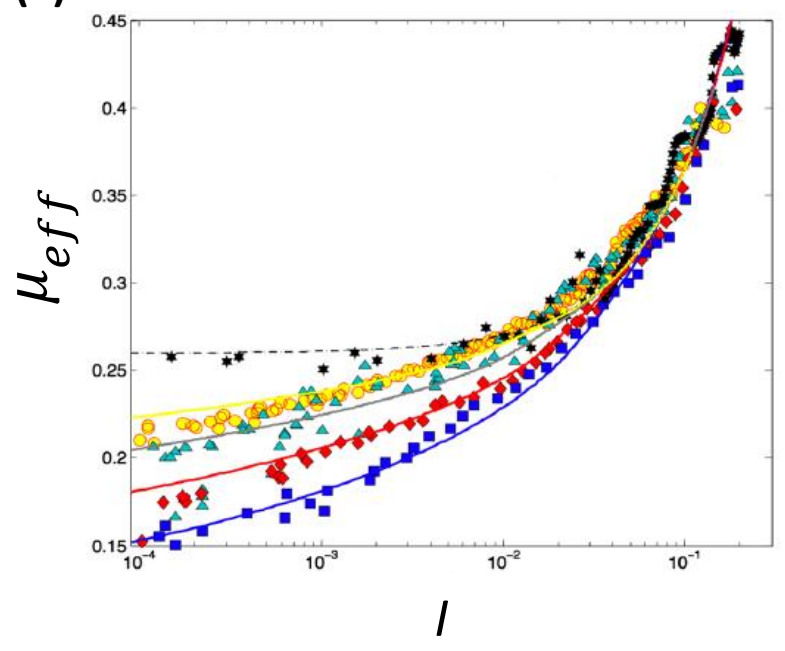

(b)

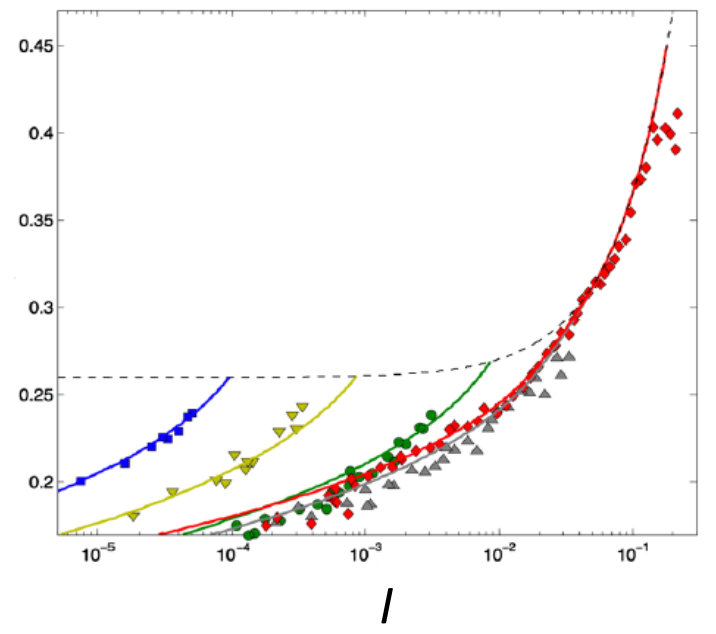

Figure 12: Non-local rheological corrections in quasi-static flows. $\mu_{\mathrm{eff}}-I$ correlations in a steady annular shear rotational drum (a) at different size ratio $R / d_{p}$ from 25 to 200 , and (b) at different shear rate of the shell, $V_{\text {wall }} \sqrt{m /\left(d_{p}{ }^{2} P_{\text {wall }}\right)}$, from 0.00025 to 2.5. Non-local correlation predictions are shown as solid curves; the symbols denote the DEM results. The figure is reprinted with permission from [78]. 


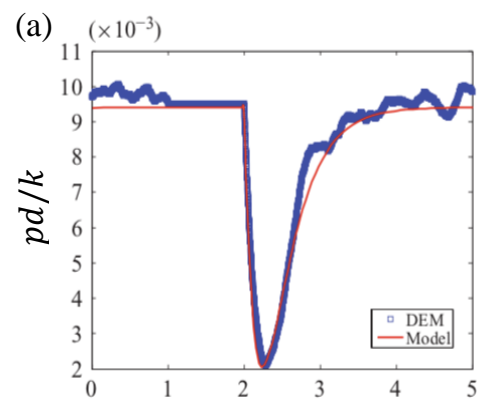

(b)

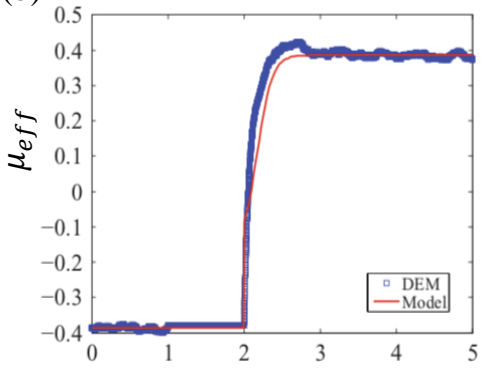

(c)
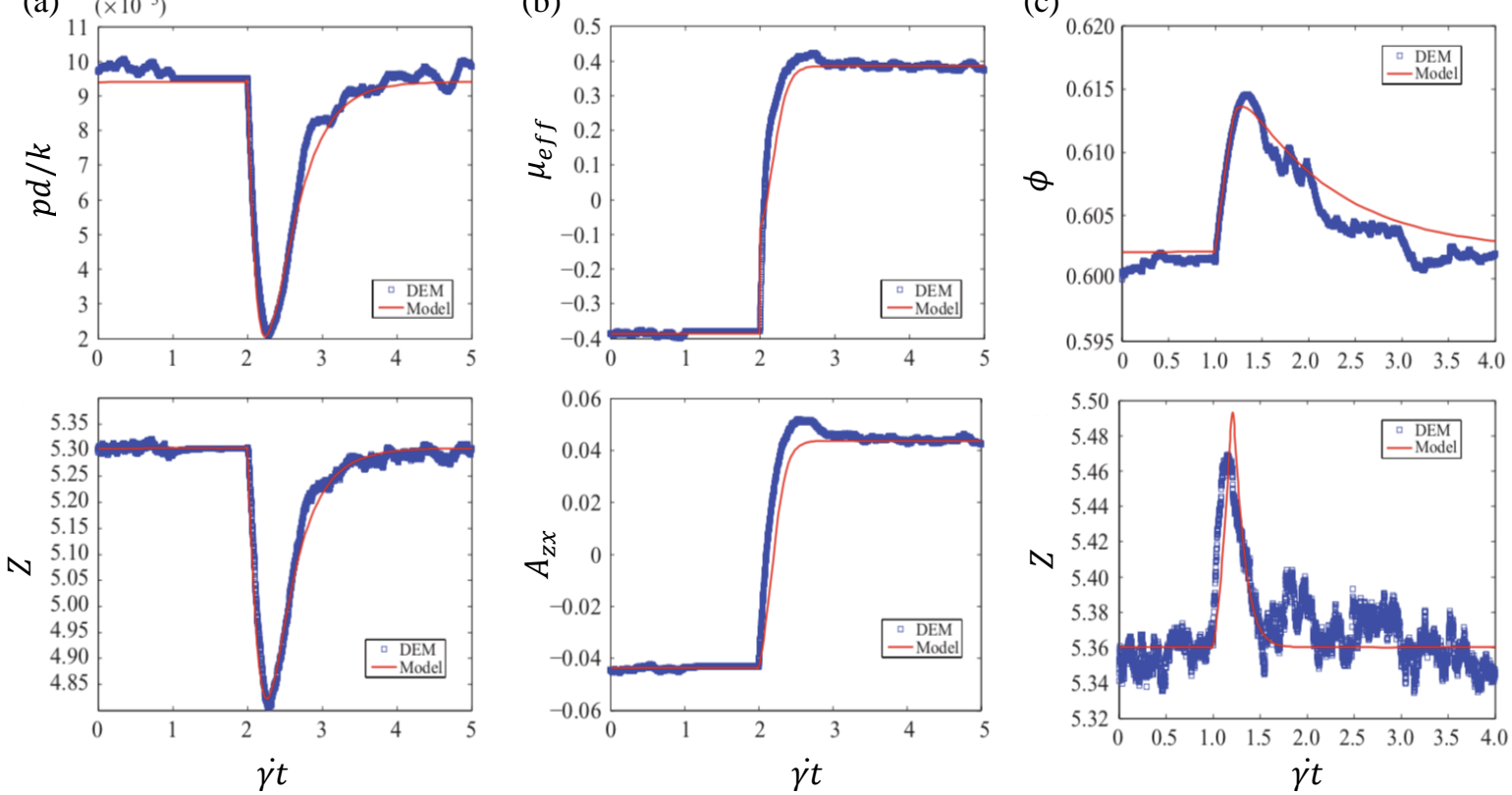

Figure 13: Evolution of (a) solid pressure and (b) effective friction coefficient under the constant volume condition, and (c) volume fraction under constant pressure conditions in comparison to the evolution of coordination number, $Z$, and $A_{\mathrm{xz}}$ for particles subjected to unsteady shear. Blue square symbols denote the DEM results, while the red solid curves are the results from constitutive models. The macroscopic friction coefficient $\mu=0.5$ and the inertia number $I \approx 0.0003$. The figure is reprinted with permission from [87]. 